\title{
Highly Luminescent Liquid Crystals in Aggregation Based on Platinum (II) Complexes
}

Xingtian $\mathrm{Hao}^{1}$, Bijin Xiong ${ }^{1}$, Mingli Ni ${ }^{1}$, Bing Tang${ }^{2}$, Ying $\mathrm{Ma}^{2}$, Haiyan Peng ${ }^{1, *}$,

Xingping Zhou ${ }^{1}$, Ivan I. Smalyukh ${ }^{3}$, Xiaolin Xie ${ }^{1, *}$

${ }^{1}$ Key Lab for Material Chemistry of Energy Conversion and Storage, Ministry of Education, and National Anti-Counterfeit Engineering Research Center, School of Chemistry and Chemical Engineering, Huazhong University of Science and Technology, Wuhan 430074, China

${ }^{2}$ State Key Laboratory of Materials Processing and Die \& Mould Technology, School of Materials Science and Engineering, Huazhong University of Science and Technology, Wuhan 430074, China

${ }^{3}$ Department of Physics and Materials Science and Engineering Program, University of Colorado at Boulder, Boulder, Colorado 80309, United States

*E-mail: hypeng@hust.edu.cn, xlxie@hust.edu.cn 


\section{Synthesis of the Tridentate Ligand.}

The tridentate ligand, namely, 2,6-bis(3-(trifluoromethyl)-1H-1,2,4-triazol-5yl)pyridine, was synthesized as Scheme S1.

Scheme S1. Schematic illustration on the synthesis of the tridentate ligand.

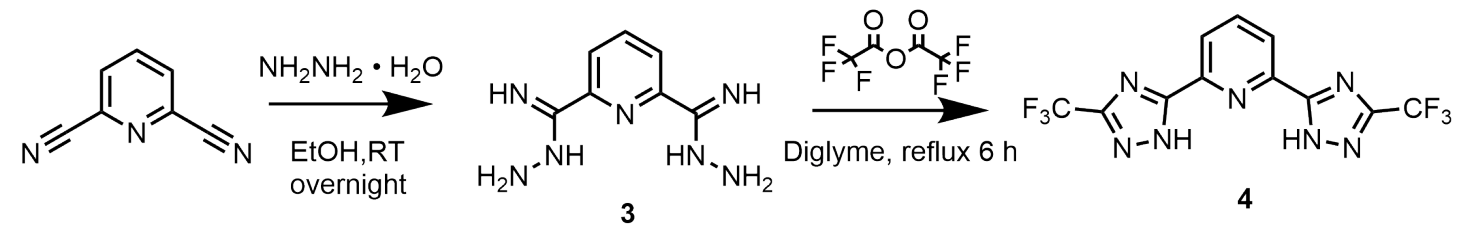

\subsection{Synthesis of pyridine-2,6-bis(carboximidhydrazide) (Compound 3).}

2,6-Pyridinedicarbonitrile $(5.00 \mathrm{~g}, 38.72 \mathrm{mmol})$ was dissolved in $100 \mathrm{~mL}$ of ethanol inside a $250 \mathrm{~mL}$ round bottom flask, followed by adding $40 \mathrm{~mL}$ of hydrazine monohydrate. The resulting solution was stirred overnight at room temperature and then filtered. The obtained yellowish solid substance was washed with ethanol for 3 times and finally dried under vacuum. Yield: 98\%.

${ }^{1} \mathrm{H}-\mathrm{NMR}\left(400 \mathrm{MHz}, \mathrm{DMSO}-d_{6}\right): \delta 7.82(\mathrm{~d}, 2 \mathrm{H}), 7.67(\mathrm{~m}, 1 \mathrm{H}), 6.09(\mathrm{~s}, 4 \mathrm{H}), 5.30(\mathrm{~s}$, $4 \mathrm{H})$.

\subsection{Synthesis of 2,6-bis(3-(trifluoromethyl)-1H-1,2,4-triazol-5-yl)pyridine}

\section{(Compound 4).}

Compound 3 (2.27 g, $11.75 \mathrm{mmol})$ was added into $30 \mathrm{~mL}$ of diethylene glycol dimethyl ether (i.e., diglyme), followed by bulk ultrasonication. Then, trifluoroacetic anhydride (5.44 g, $25.90 \mathrm{mmol}$ ) was added dropwise. During this process, the suspension changed to homogenous solution in yellow color, which was then heated to $120{ }^{\circ} \mathrm{C}$ and kept refluxing for $6 \mathrm{~h}$. After cooling down the solution to room temperature, $70 \mathrm{~mL}$ of 
distilled water and $2 \mathrm{~mL}$ of concentrated $\mathrm{HCl}$ were added sequentially. Subsequently, the mixture was heated to $90^{\circ} \mathrm{C}$ and kept reflux overnight. The solution was then cooled down to room temperature and continuously stirred until the white precipitate appeared. The white precipitate was filtered and sequentially washed with water and petroleum ether. The final product was dried overnight under vacuum at $80{ }^{\circ} \mathrm{C}$. Yield: $75 \%$.

${ }^{1} \mathrm{H}-\mathrm{NMR}\left(400 \mathrm{MHz}, \mathrm{DMSO}-d_{6}\right): \delta 8.29-8.22(\mathrm{~m})$

${ }^{19}$ F-NMR (600 MHz, DMSO- $\left.d_{6}\right): \delta-63.80$.

ESI-HR-MS (positive scan, m/z): $[\mathrm{M}+\mathrm{H}]^{+}$calculated 350.0583; found 350.0586.

\section{Synthesis of Cis-Dichlorobis(dimethylsulfoxide)platinum (II).}

Cis-Dichlorobis(dimethylsulfoxide)platinum (II) (i.e., $\mathrm{PtCl}_{2}(\mathrm{DMSO})_{2}$ ) was synthesized as Scheme S2.

Scheme S2. Schematic illustration on the synthesis of $\mathrm{PtCl}_{2}(\mathrm{DMSO})_{2}$.

$$
\mathrm{K}_{2} \mathrm{PtCl}_{4}+2 \mathrm{DMSO} \longrightarrow \mathrm{PtCl}_{2}(\mathrm{DMSO})_{2}+2 \mathrm{KCl}
$$

Potassium tetrachloroplatinate (II) $(1.24 \mathrm{~g}, 3.00 \mathrm{mmol})$ was dissolved in $10 \mathrm{~mL}$ of deionized water and filtered to remove the impurities (e.g., metallic $\mathrm{Pt}, \mathrm{K}_{2} \mathrm{PtCl}_{6}$ ). Then, DMSO (0.64 mL, $9.00 \mathrm{mmol})$ was added to form a homogeneous solution, which was continuously stirred for $12 \mathrm{~h}$ at room temperature. The generated precipitate during reaction was filtered and sequentially washed with water, ethanol and diethyl ether. The product was dried overnight under vacuum. Yield: 90\%.

Elemental analysis for $c i s-\left[\mathrm{PtCl}_{2}(\mathrm{DMSO})_{2}\right]$, calculated: C, $11.38 \% ; \mathrm{H}, 2.86 \%$; 15.19\%. Found: C, 11.28\%; H, 2.80\%; S, 15.36\%. 


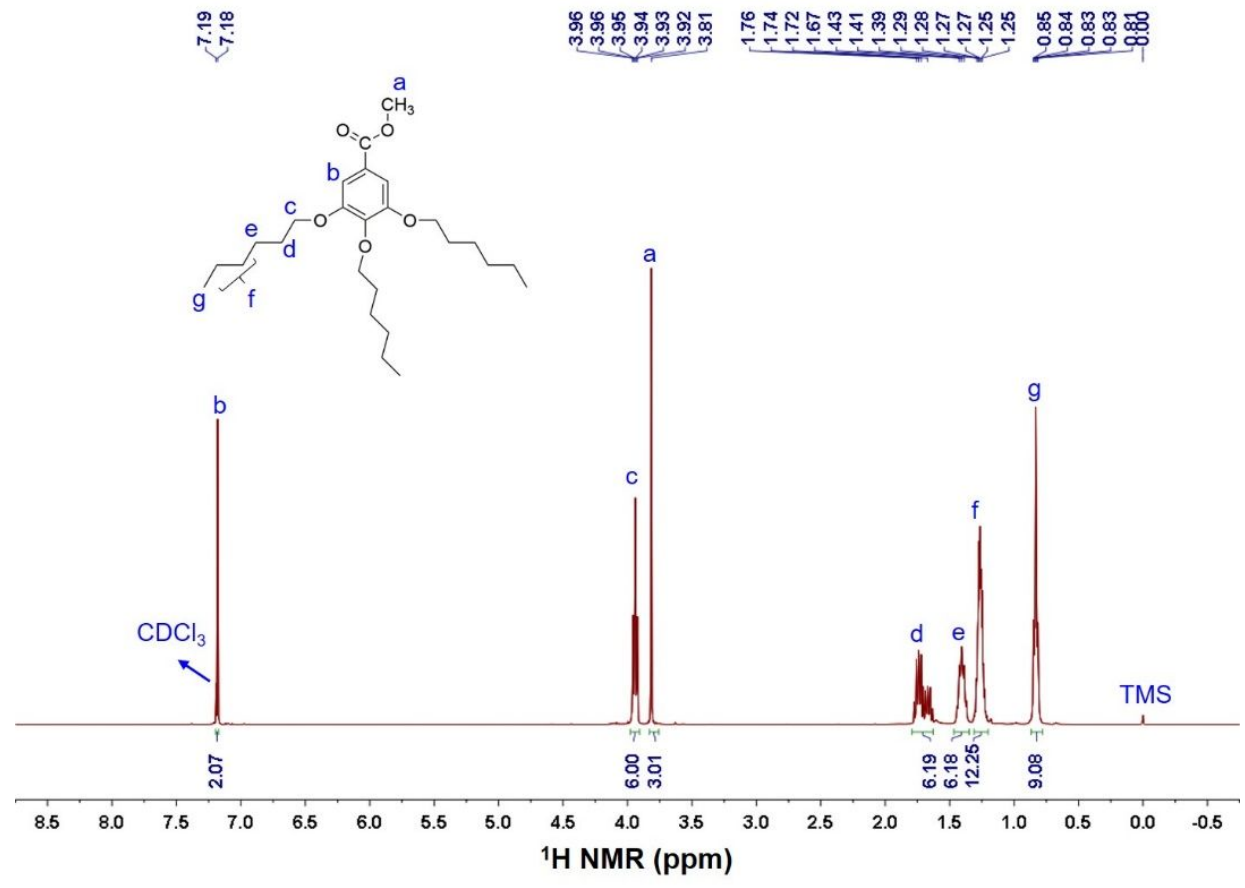

Figure S1. ${ }^{1} \mathrm{H}-\mathrm{NMR}$ spectrum of $1 \mathrm{a}$ in $\mathrm{CDCl}_{3}$

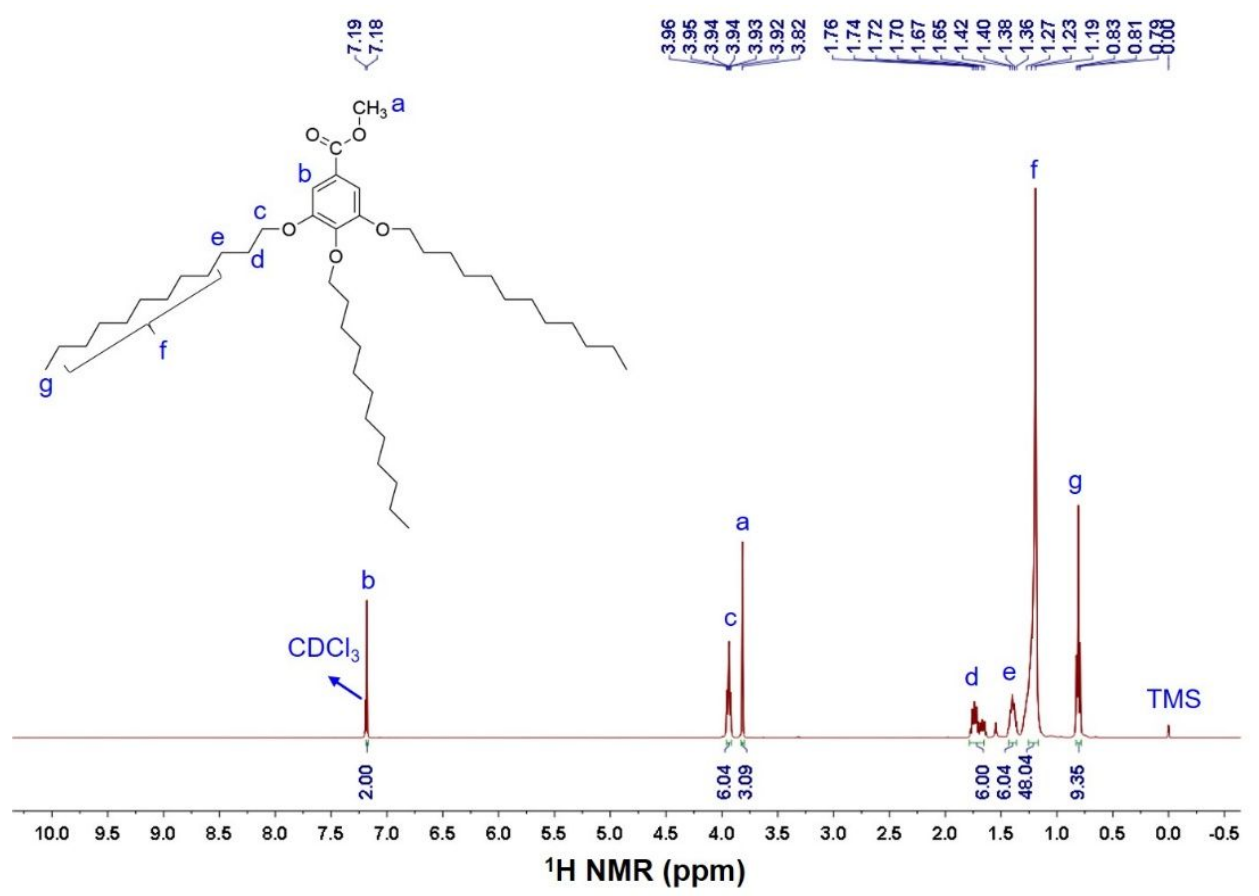

Figure S2. ${ }^{1} \mathrm{H}-\mathrm{NMR}$ spectrum of $\mathbf{1 b}$ in $\mathrm{CDCl}_{3}$ 


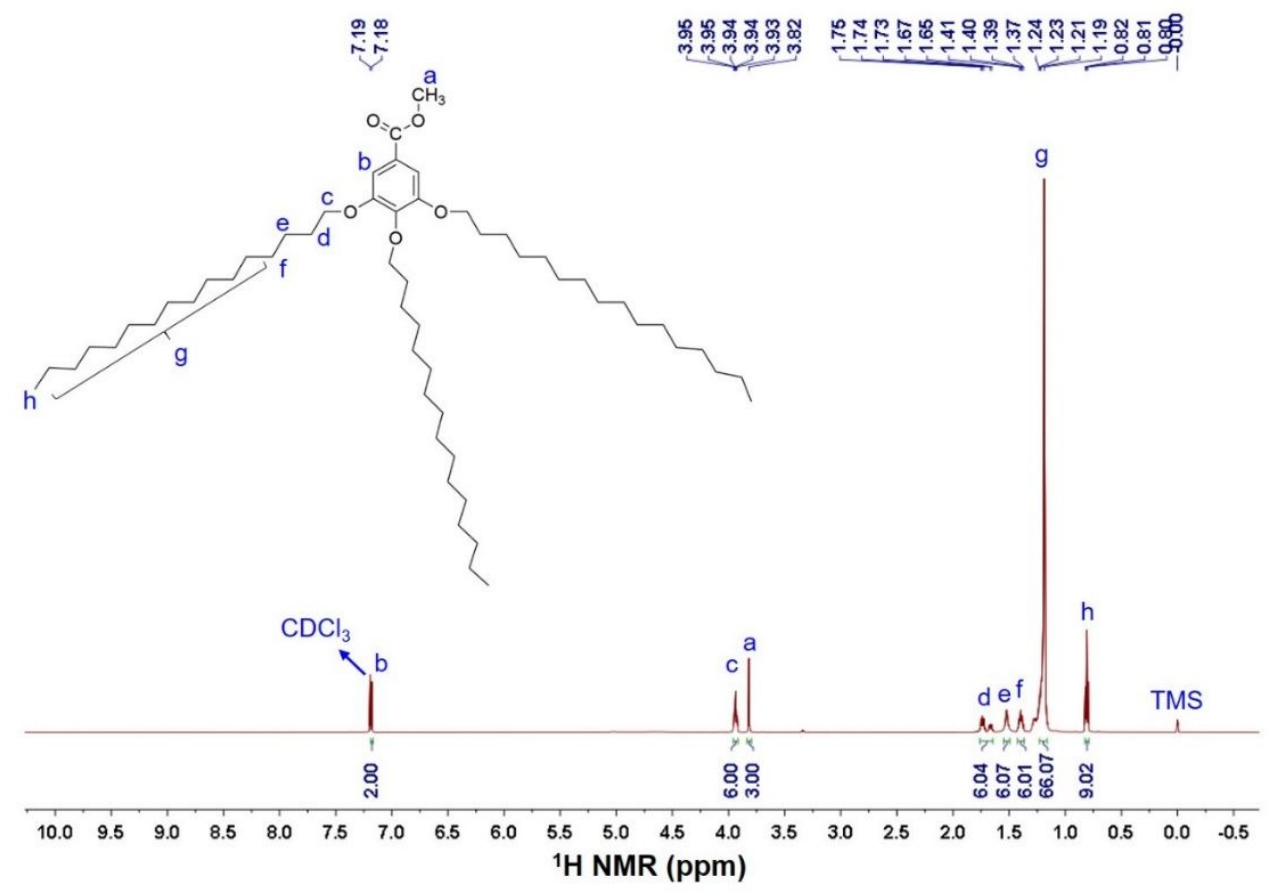

Figure S3. ${ }^{1} \mathrm{H}-\mathrm{NMR}$ spectrum of $1 \mathrm{c}$ in $\mathrm{CDCl}_{3}$

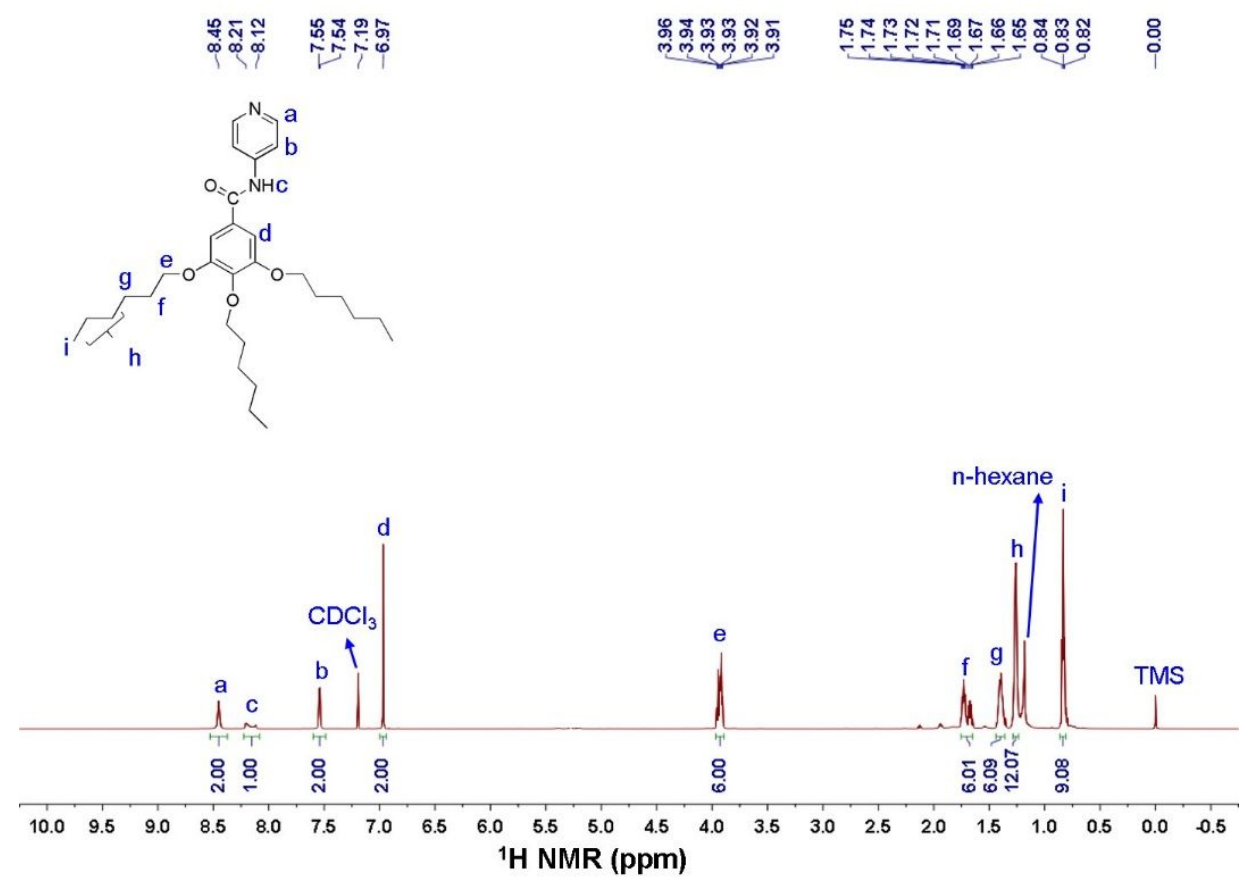

Figure S4. ${ }^{1} \mathrm{H}-\mathrm{NMR}$ spectrum of $\mathbf{2 a}$ in $\mathrm{CDCl}_{3}$ 


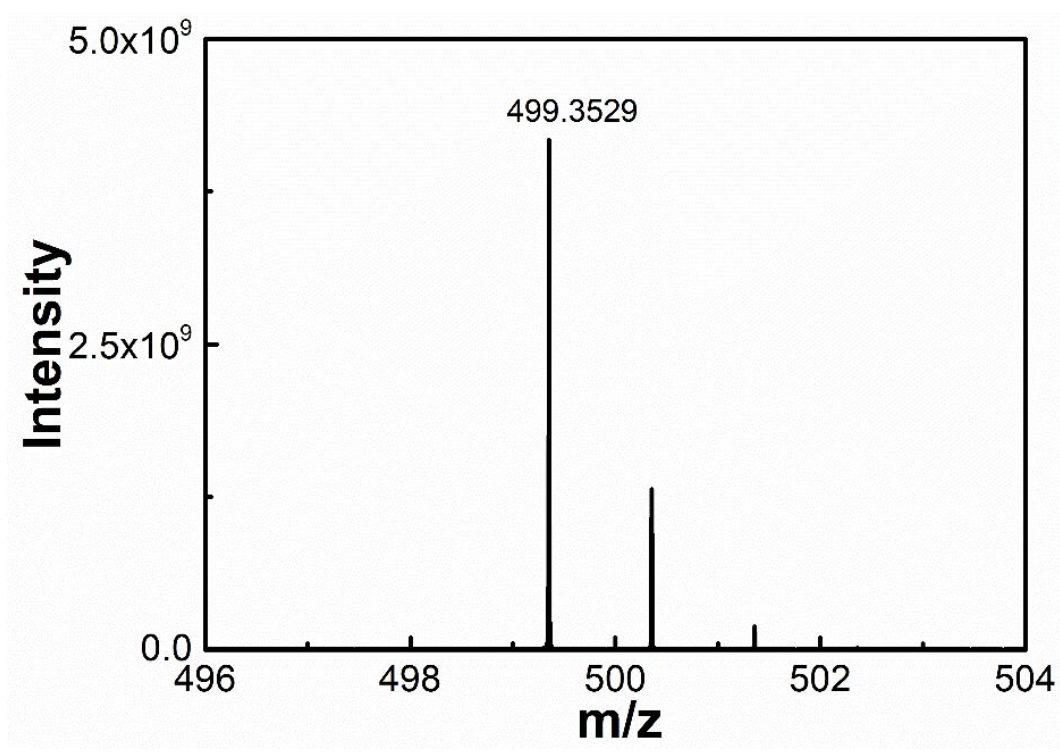

Figure S5. ESI-HR-MS of $\mathbf{2 a}$

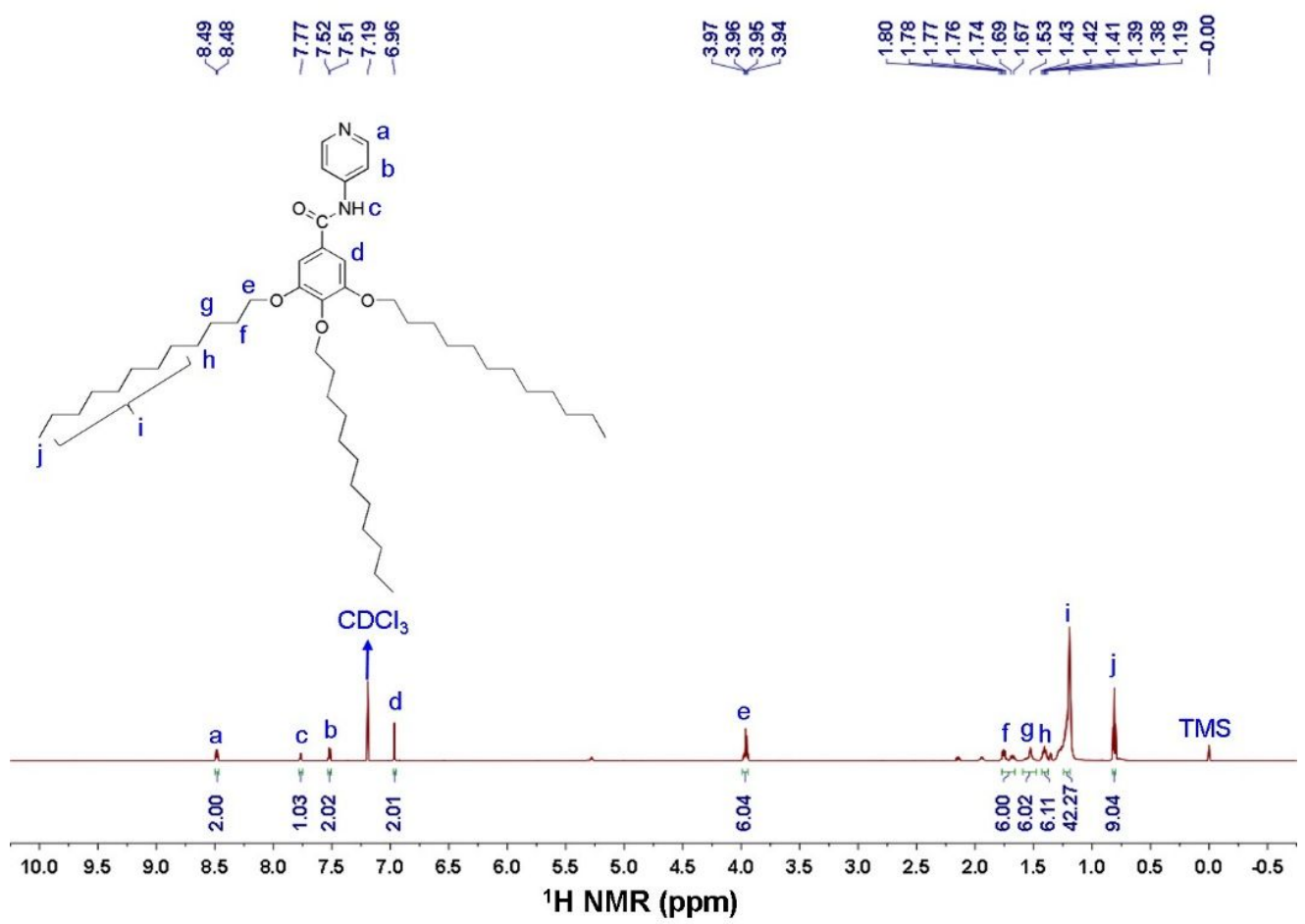

Figure S6. ${ }^{1} \mathrm{H}-\mathrm{NMR}$ spectrum of $\mathbf{2 b}$ in $\mathrm{CDCl}_{3}$ 


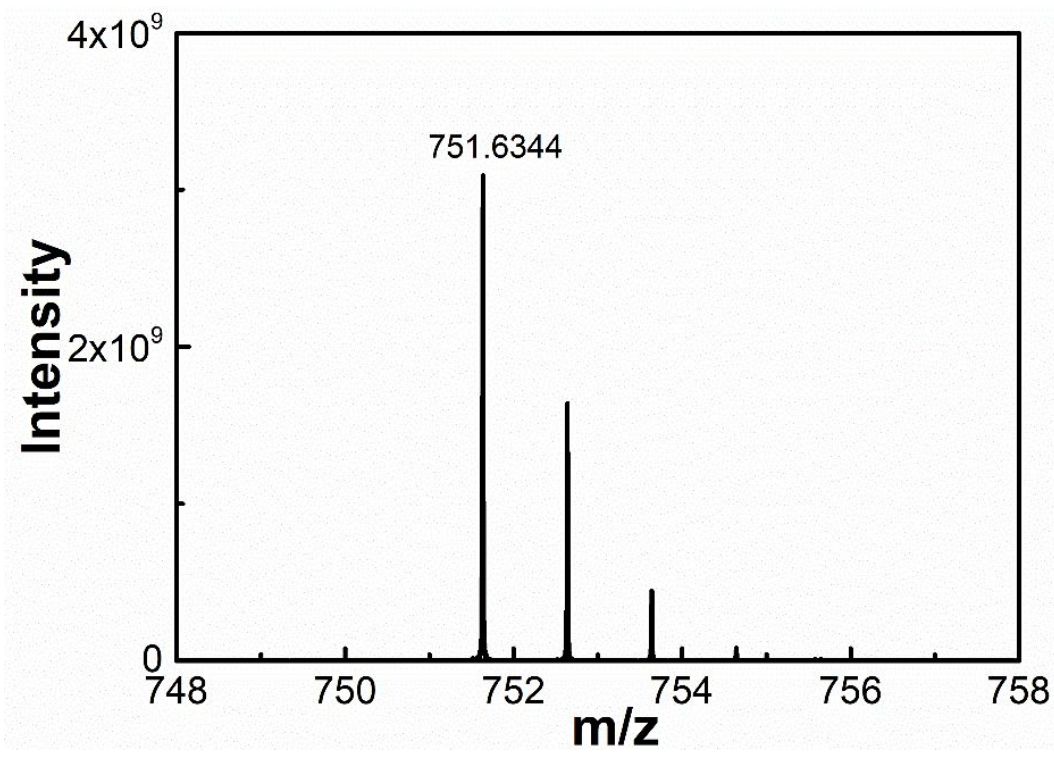

Figure S7. ESI-HR-MS of $\mathbf{2 b}$

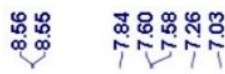

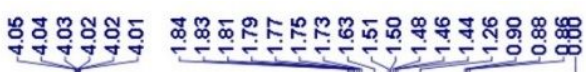

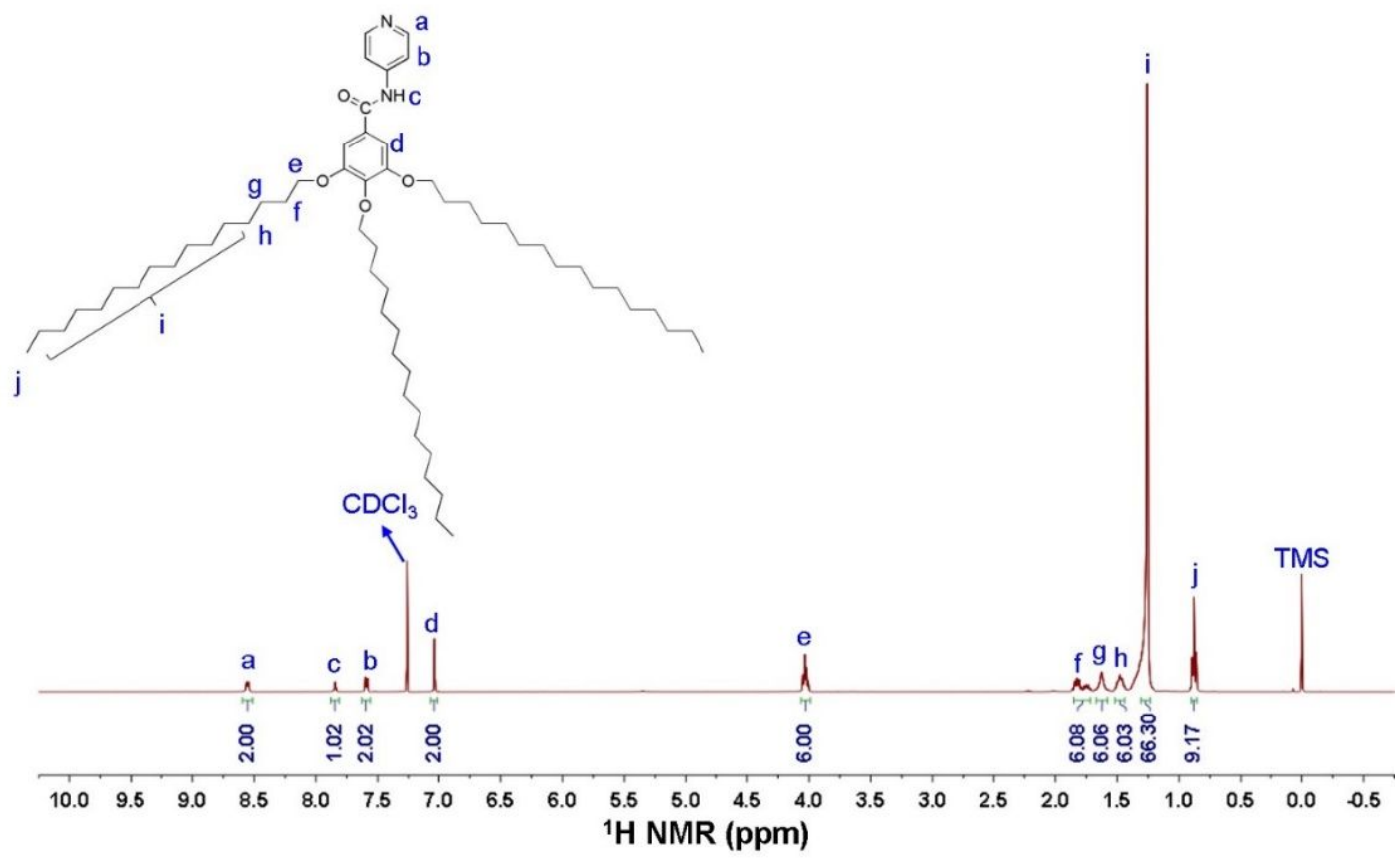

Figure S8. ${ }^{1} \mathrm{H}-\mathrm{NMR}$ spectrum of $2 \mathrm{c}$ in $\mathrm{CDCl}_{3}$ 


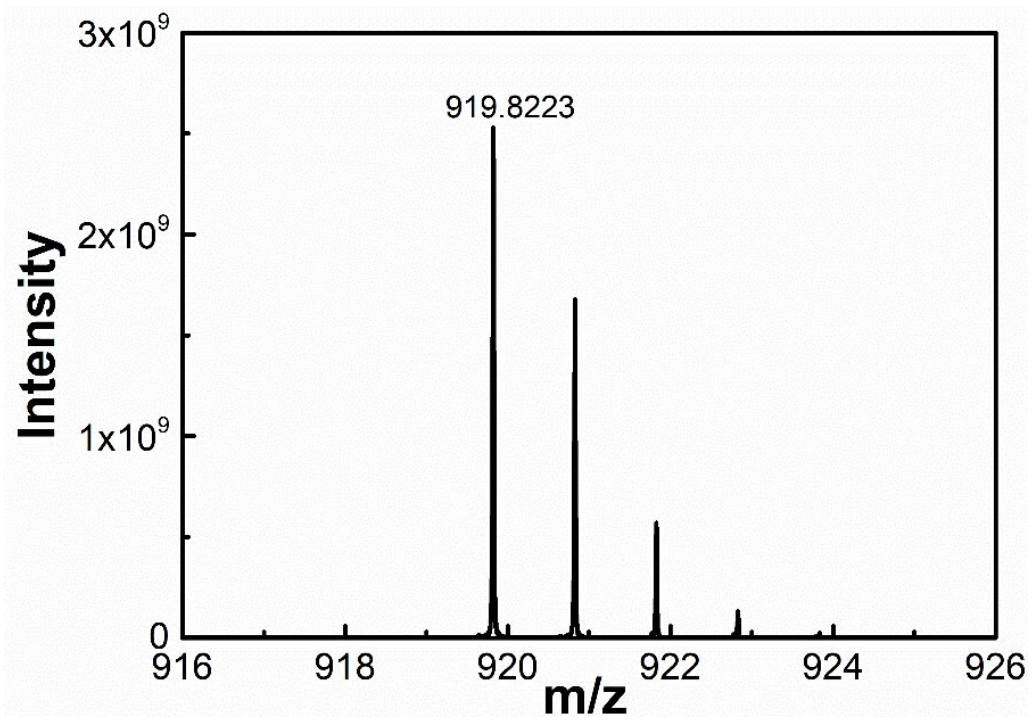

Figure S9. ESI-HR-MS of 2c

\begin{tabular}{|c|c|}
\hline 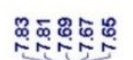 & 8 \\
\hline
\end{tabular}
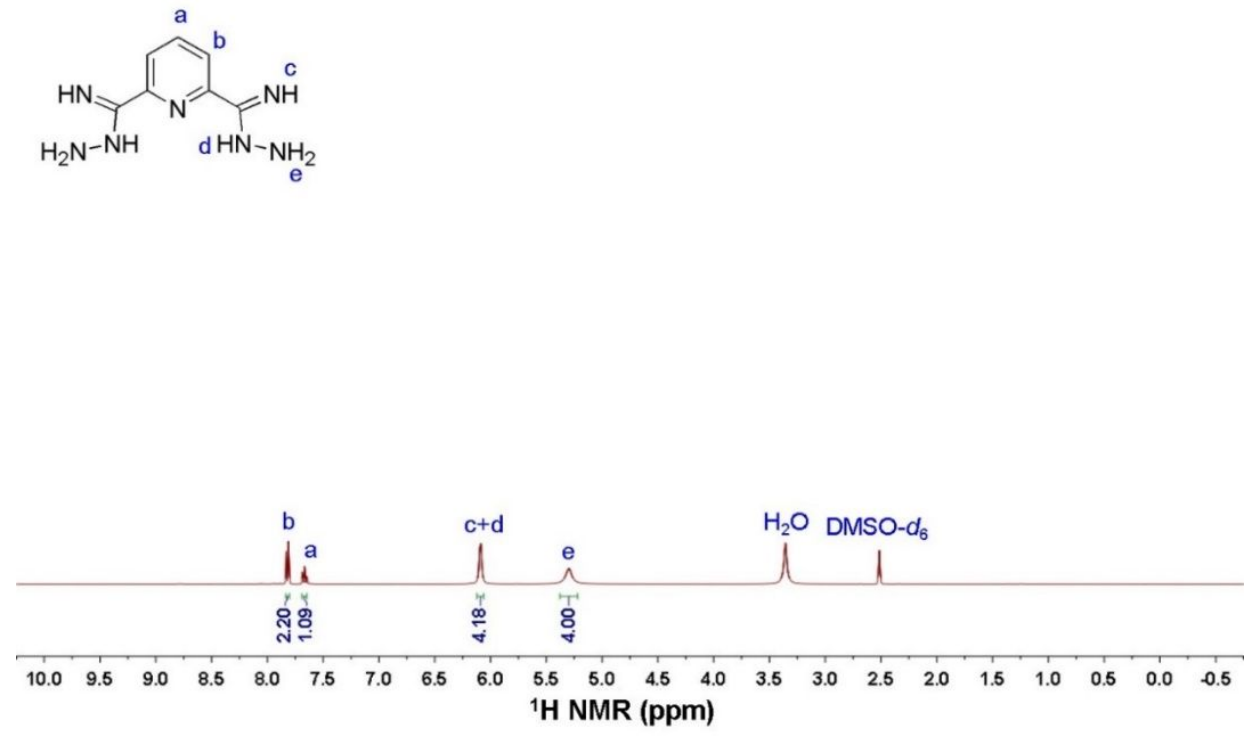

Figure S10. ${ }^{1} \mathrm{H}-\mathrm{NMR}$ spectrum of $\mathbf{3}$ in DMSO- $d_{6}$ 


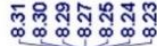

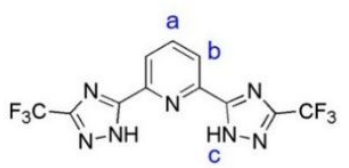

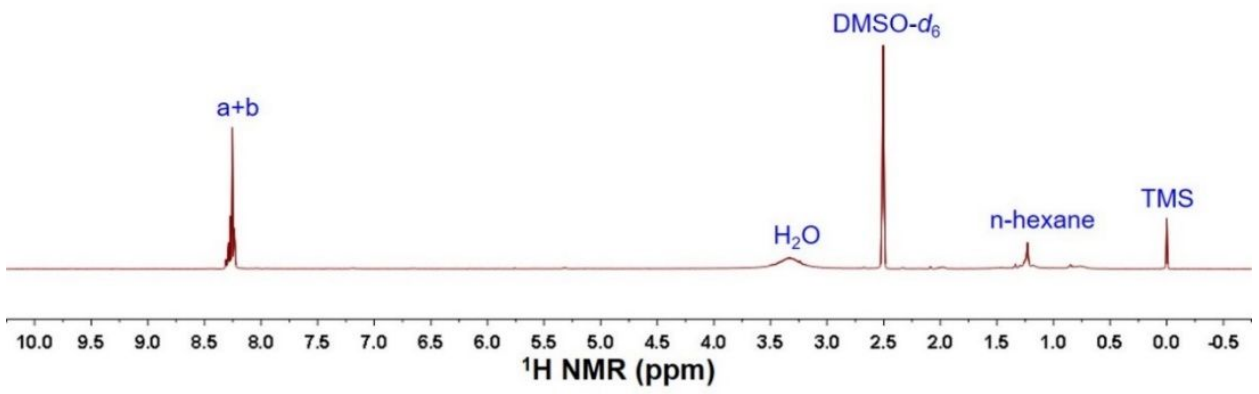

Figure S11. ${ }^{1} \mathrm{H}-\mathrm{NMR}$ spectrum of 4 in DMSO- $d_{6}$

Note: Proton on the $\mathrm{N}$ atom was not detected.
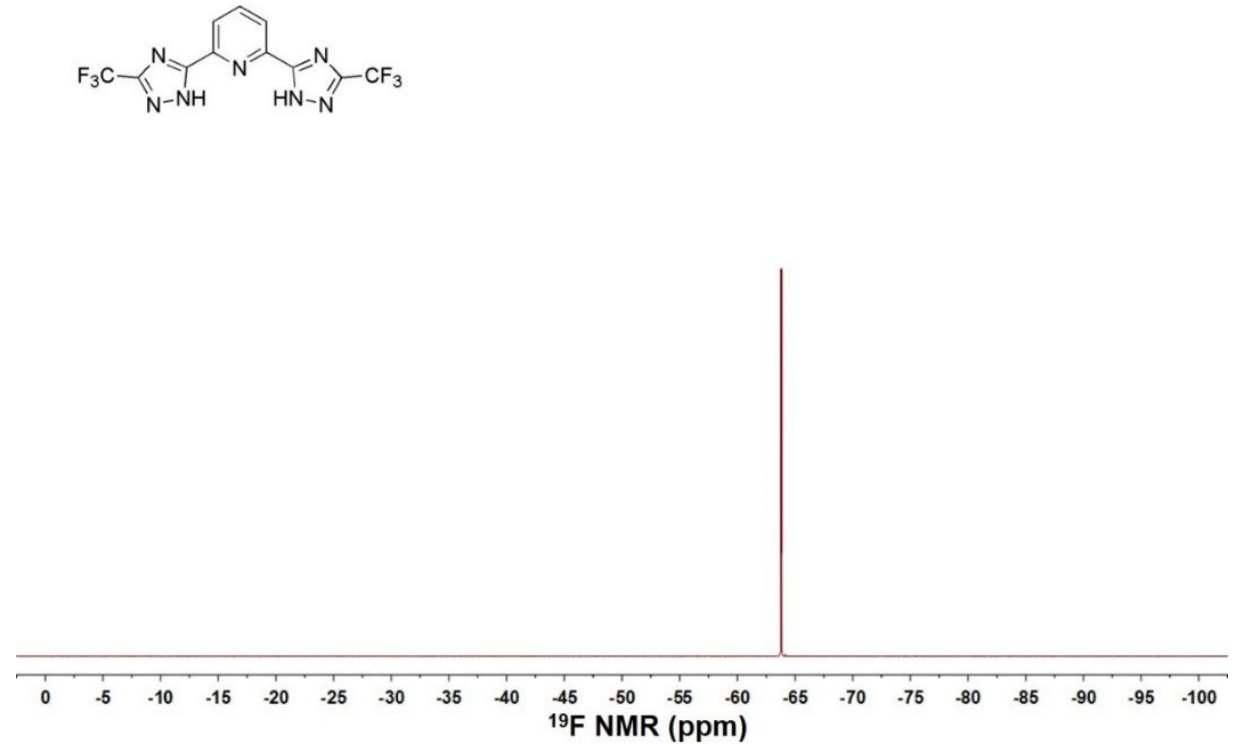

Figure S12. ${ }^{19} \mathrm{~F}-\mathrm{NMR}$ spectrum of $\mathbf{4}$ in DMSO- $d_{6}$ 


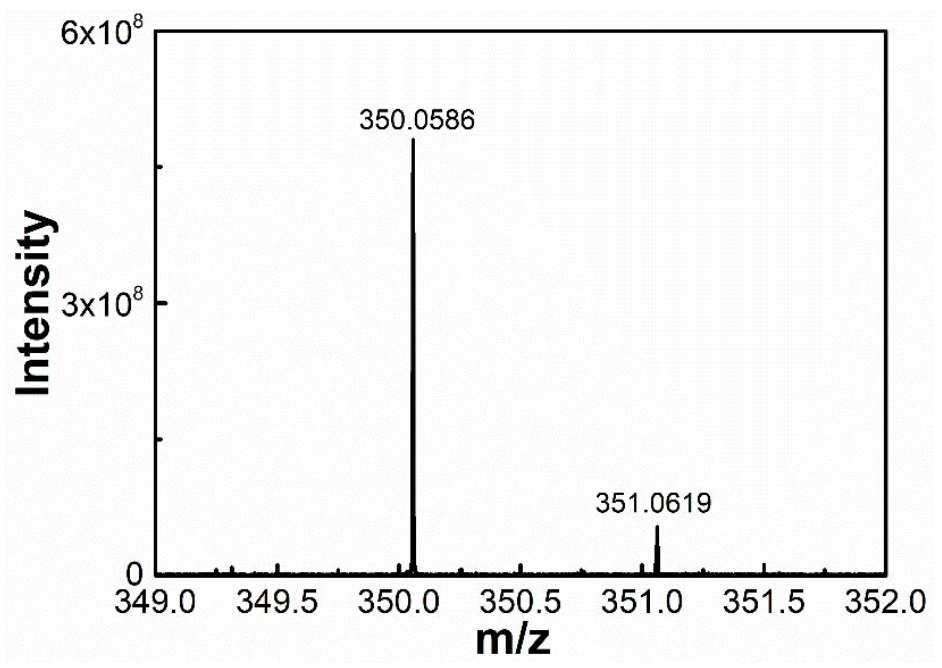

Figure S13. ESI-HR-MS of 4

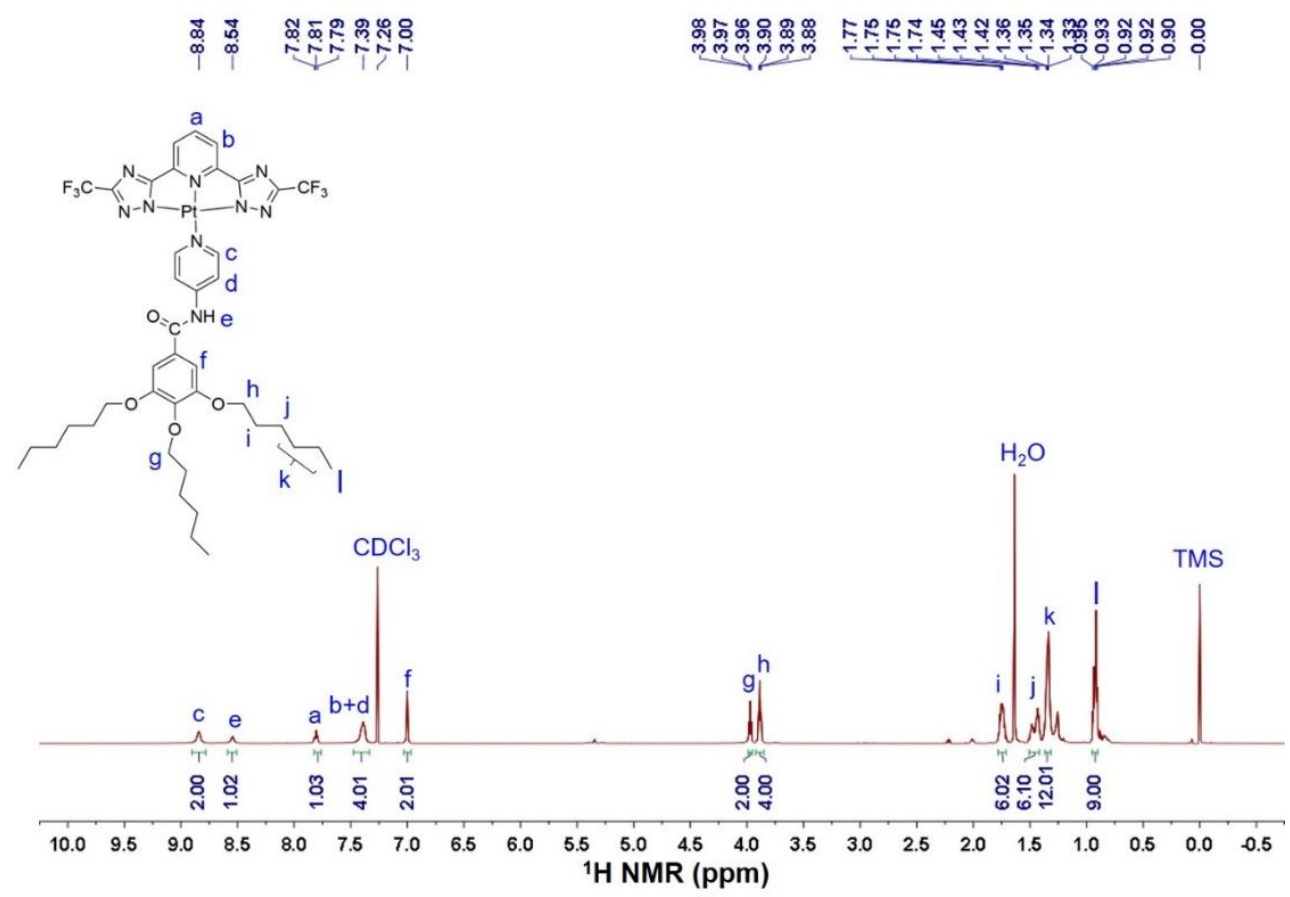

Figure S14. ${ }^{1} \mathrm{H}-\mathrm{NMR}$ spectrum of the complex $\mathbf{P t}-\mathbf{3 O C} \mathbf{C}_{\mathbf{6}} \mathbf{H}_{13}$ in $\mathrm{CDCl}_{3}$ 


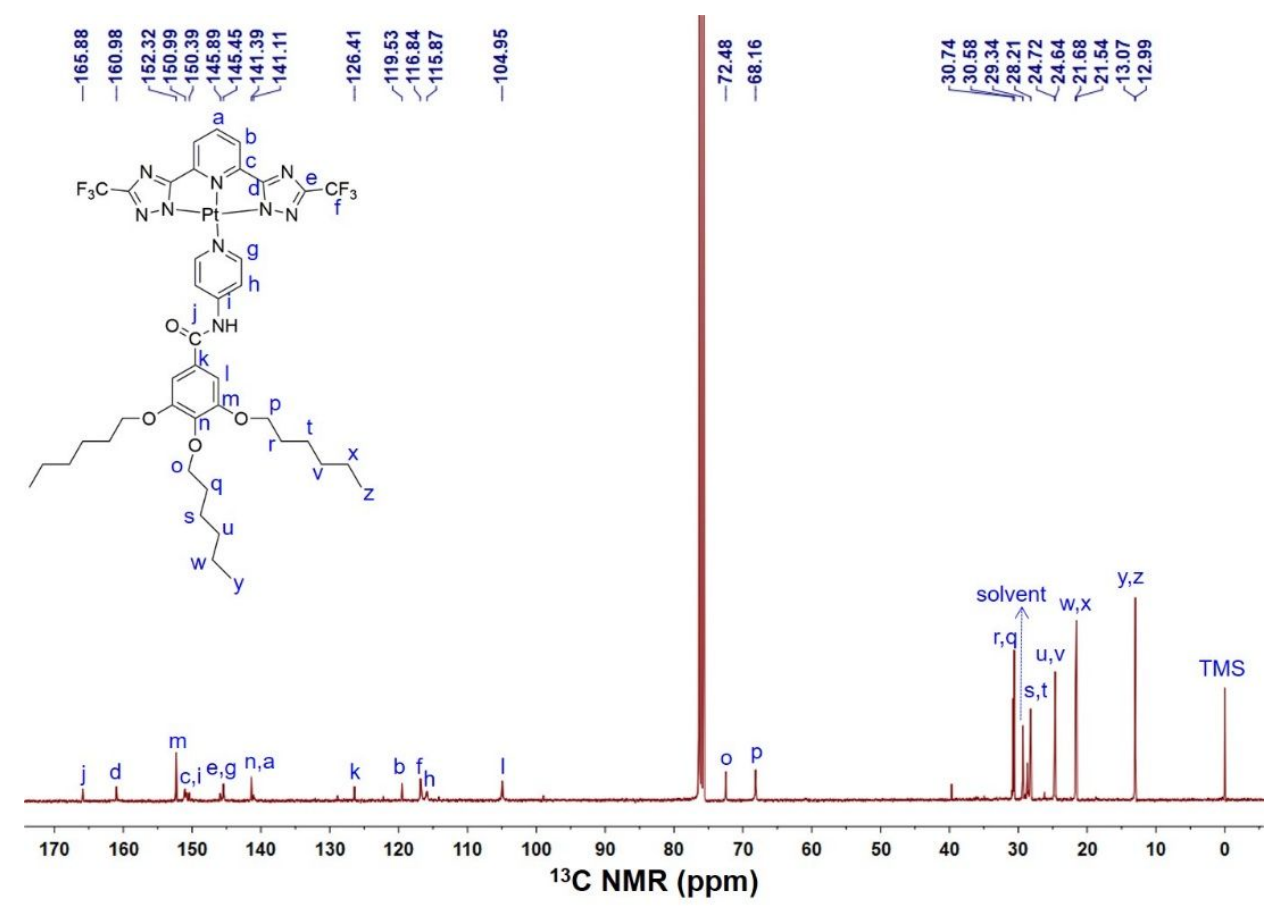

Figure S15. ${ }^{13} \mathrm{C}-\mathrm{NMR}$ spectrum of the complex $\mathbf{P t}-\mathbf{3 O C} \mathbf{C}_{6} \mathbf{H}_{13}$ in $\mathrm{CDCl}_{3}$

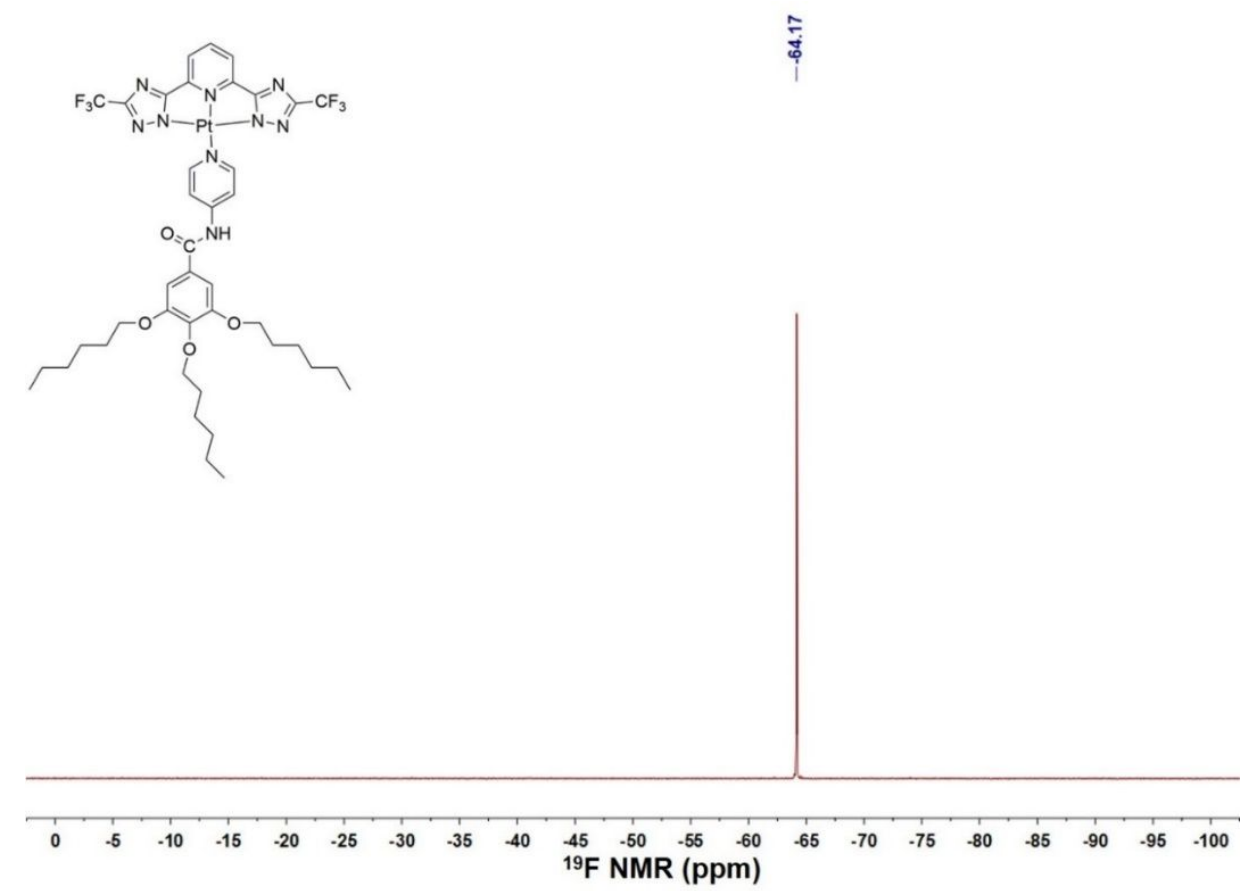

Figure S16. ${ }^{19}$ F-NMR spectrum of the complex Pt-3OC $\mathbf{C H}_{\mathbf{6}}$ in $\mathrm{CDCl}_{3}$ 


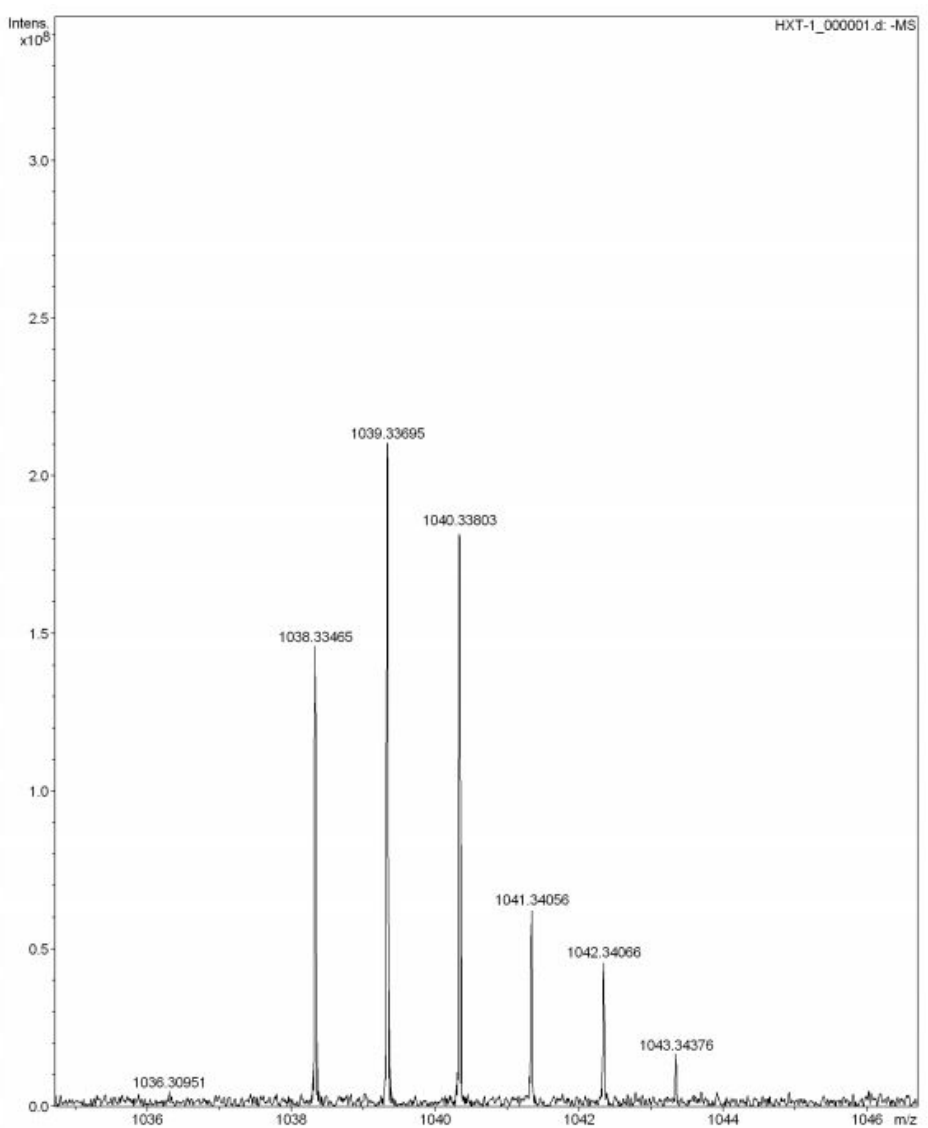

Figure S17. ESI-HR-MS of the complex Pt-3OC ${ }_{6} \mathbf{H}_{13}$

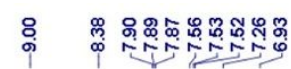

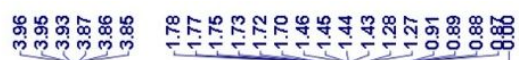
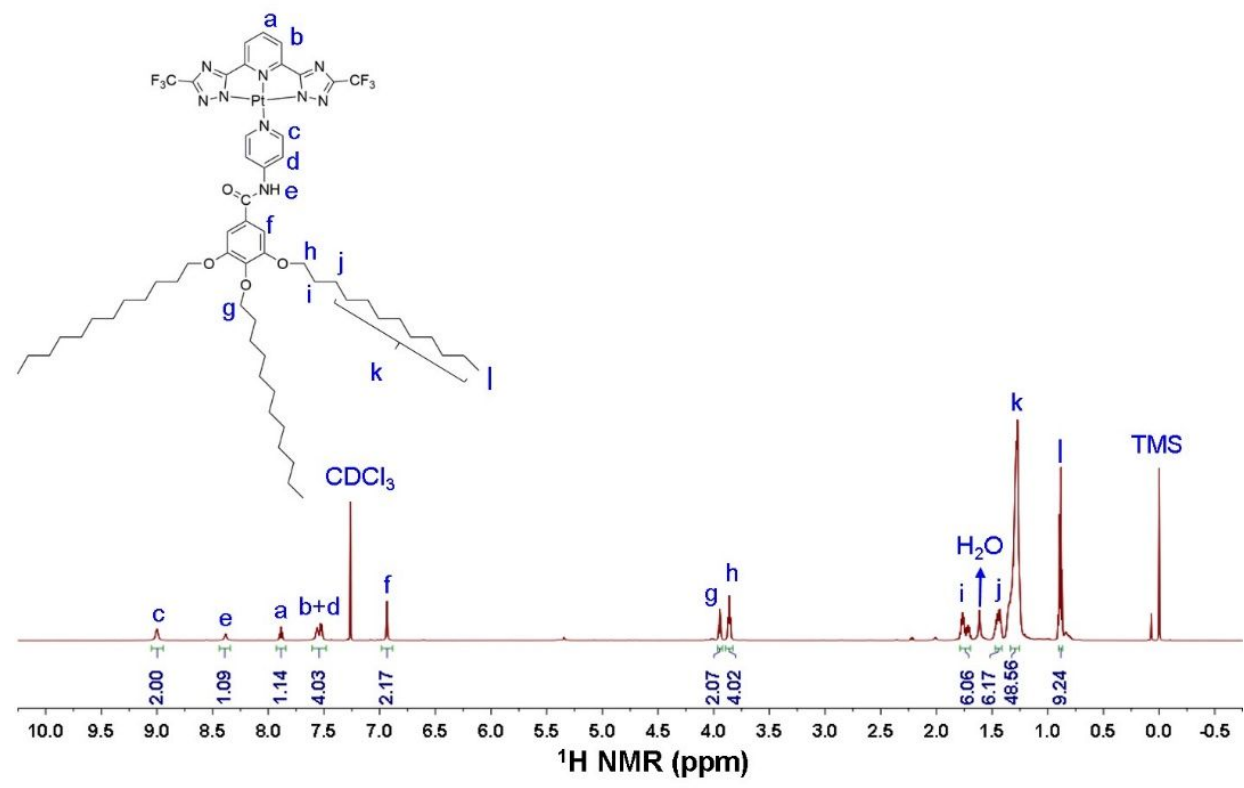

Figure S18. ${ }^{1} \mathrm{H}-\mathrm{NMR}$ spectrum of the complex $\mathbf{P t}-\mathbf{3 O C} \mathbf{1 2}_{\mathbf{1 2}} \mathbf{H}_{\mathbf{2}}$ in $\mathrm{CDCl}_{3}$ 


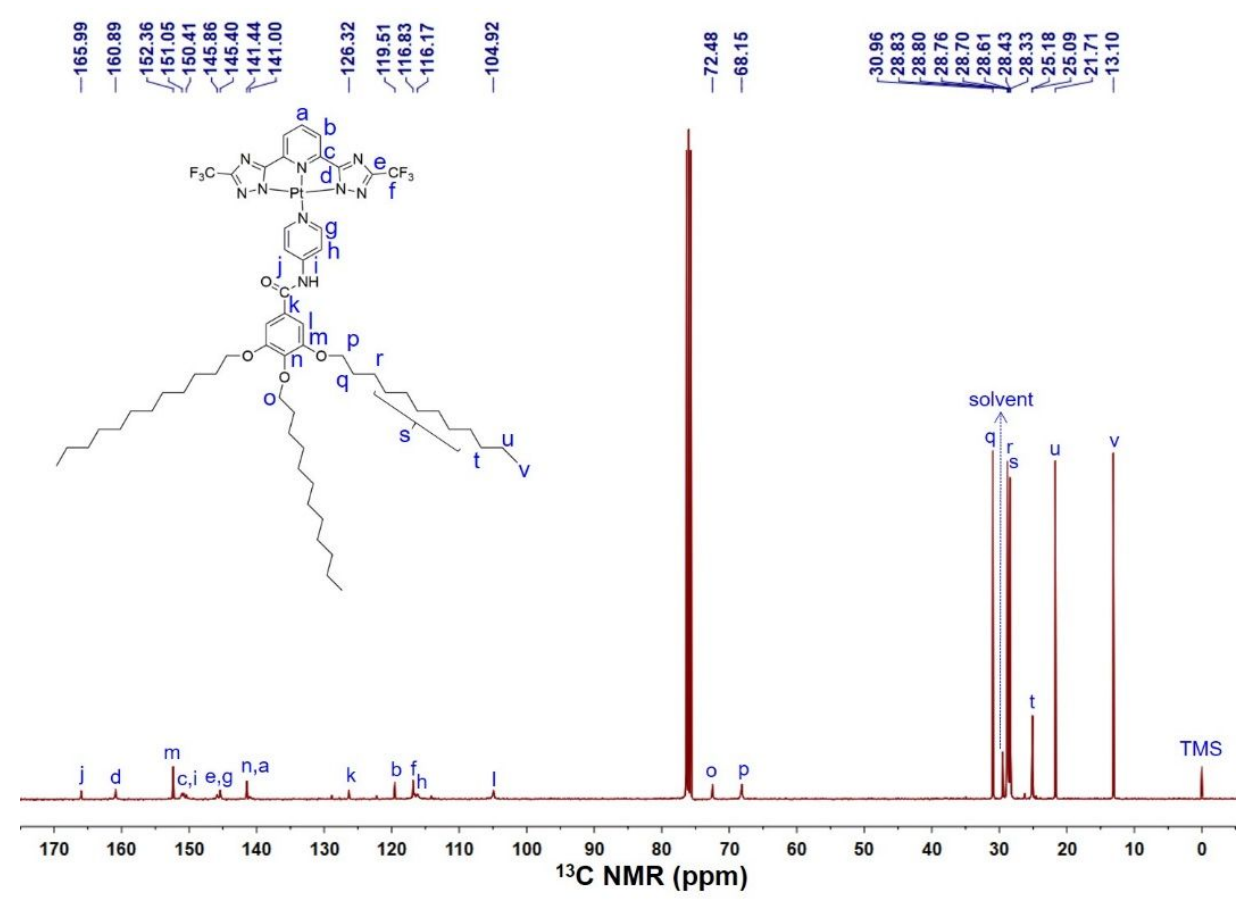

Figure S19. ${ }^{13} \mathrm{C}-\mathrm{NMR}$ spectrum of the complex $\mathbf{P t}-\mathbf{3 O C}_{\mathbf{1 2}} \mathbf{H}_{\mathbf{2 5}}$ in $\mathrm{CDCl}_{3}$

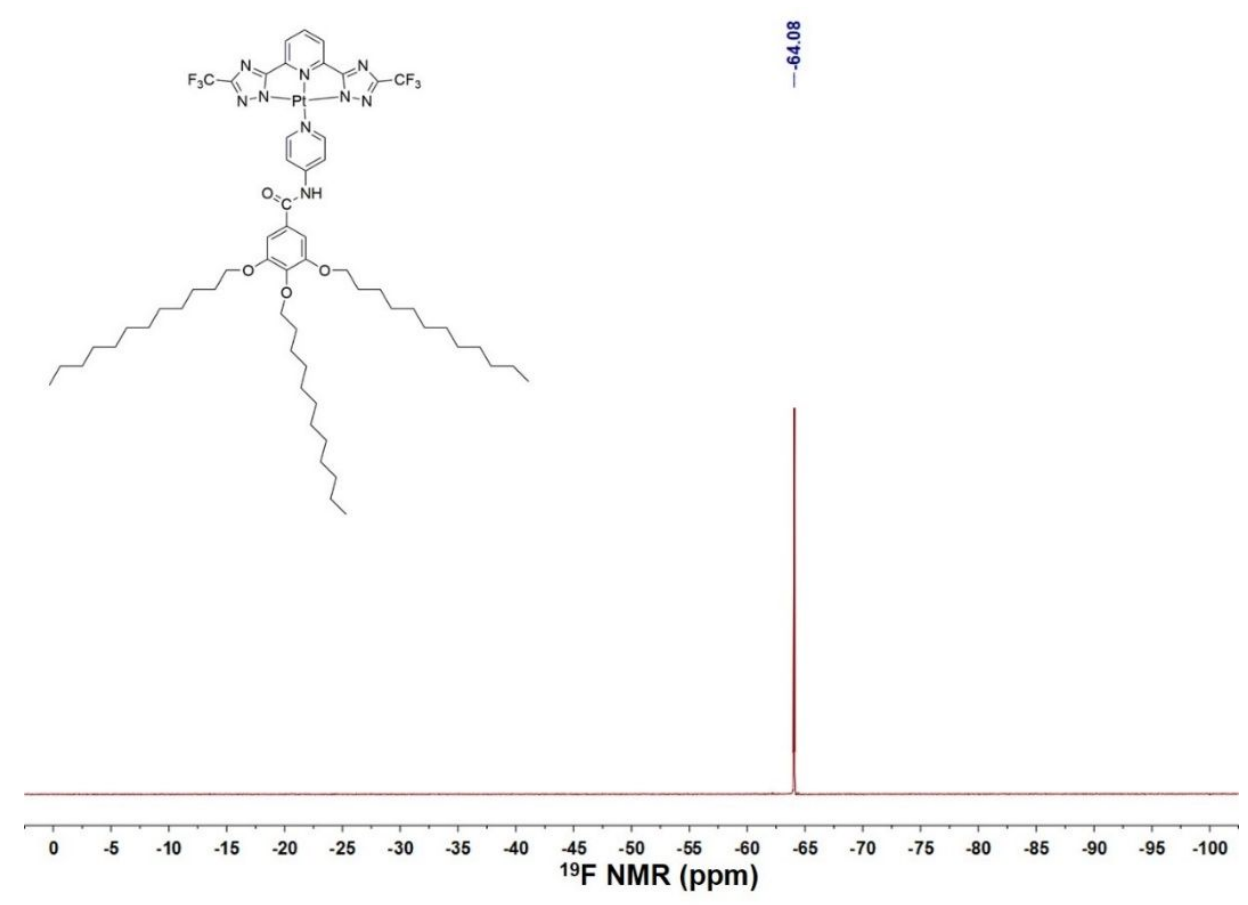

Figure S20. ${ }^{19}$ F-NMR spectrum of the complex $\mathbf{P t}-\mathbf{3 O C}_{\mathbf{1 2}} \mathbf{H}_{\mathbf{2}}$ in $\mathrm{CDCl}_{3}$ 


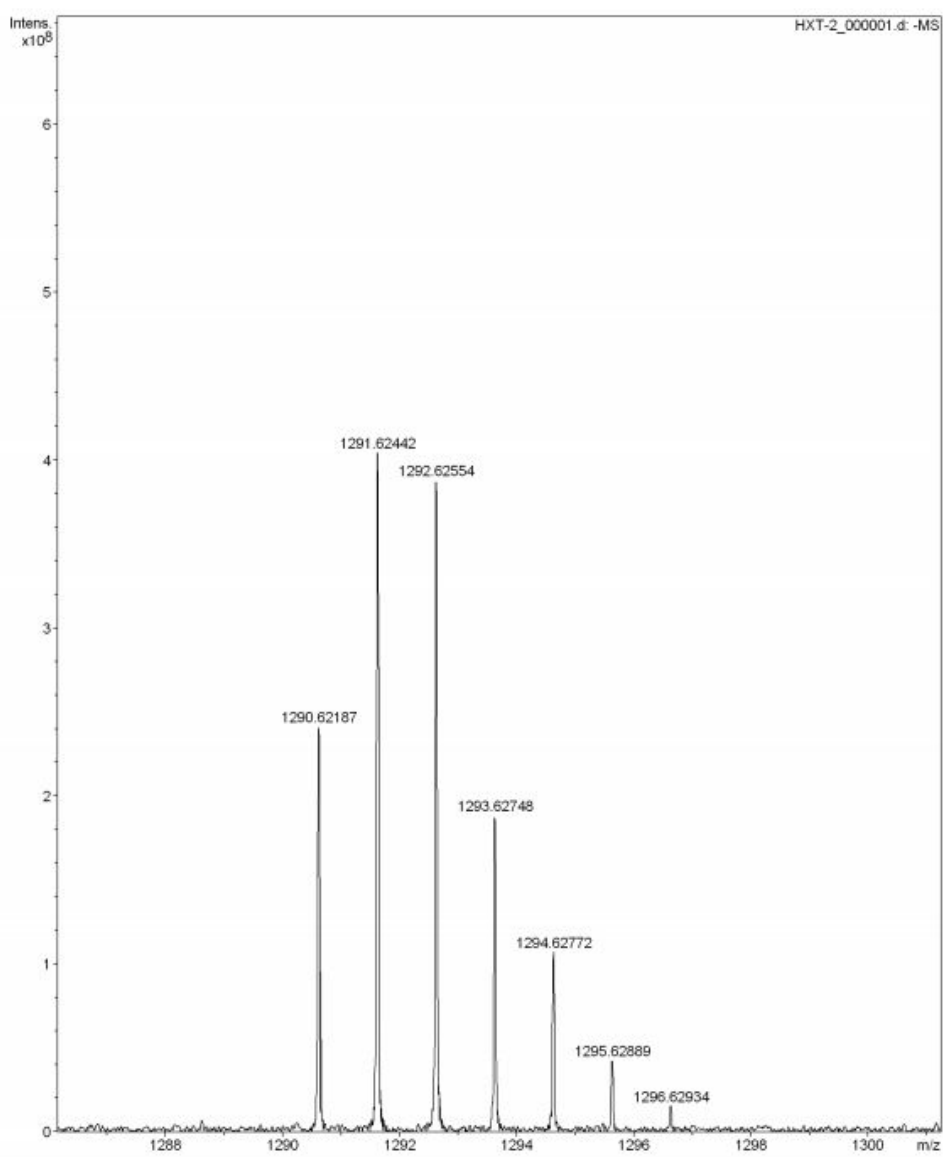

Figure S21. ESI-HR-MS of the complex Pt-3OC $\mathbf{C H}_{\mathbf{1 2}} \mathbf{H}_{25}$

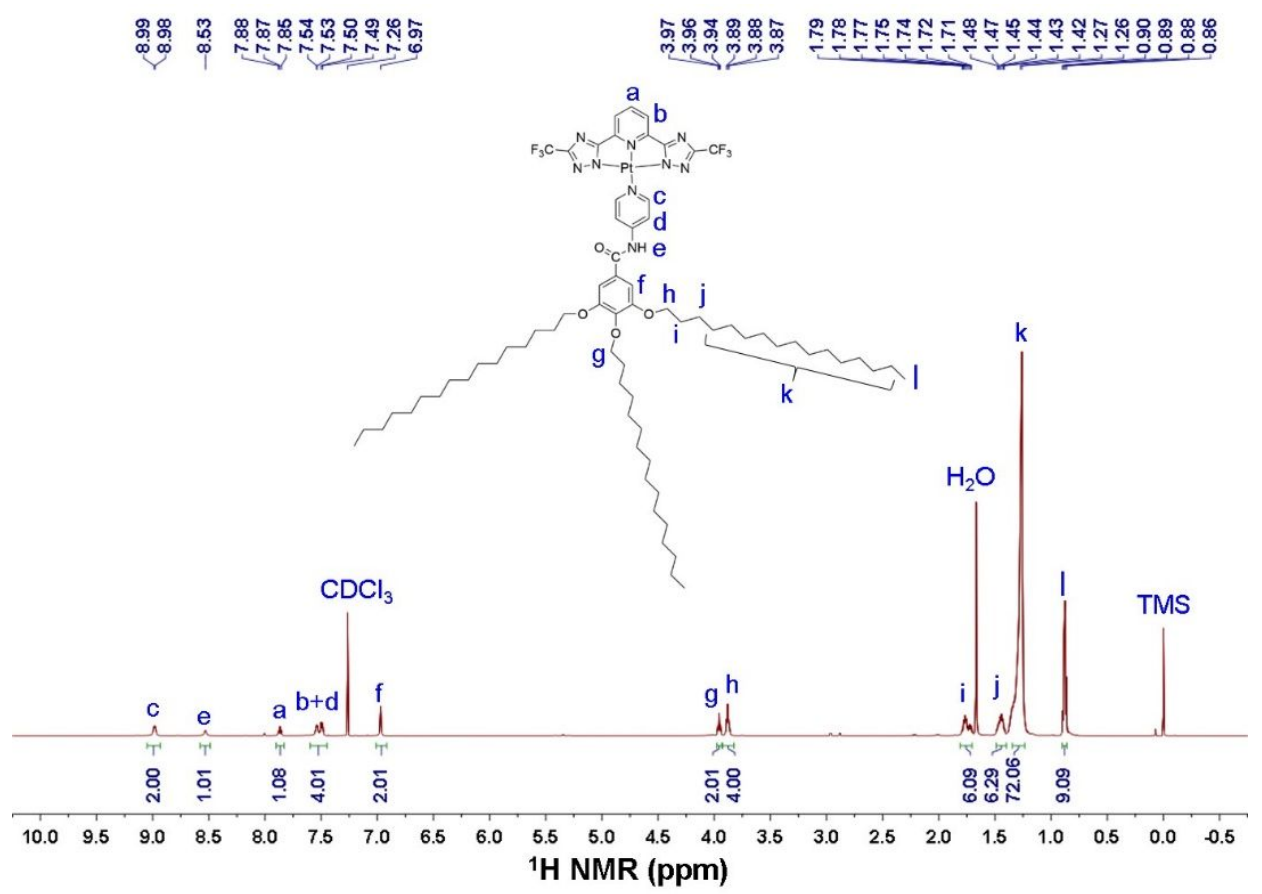

Figure S22. ${ }^{1} \mathrm{H}-\mathrm{NMR}$ spectrum of the complex $\mathbf{P t}-\mathbf{3 O C} \mathbf{C}_{\mathbf{1 6}} \mathbf{H}_{\mathbf{3 3}}$ in $\mathrm{CDCl}_{3}$ 


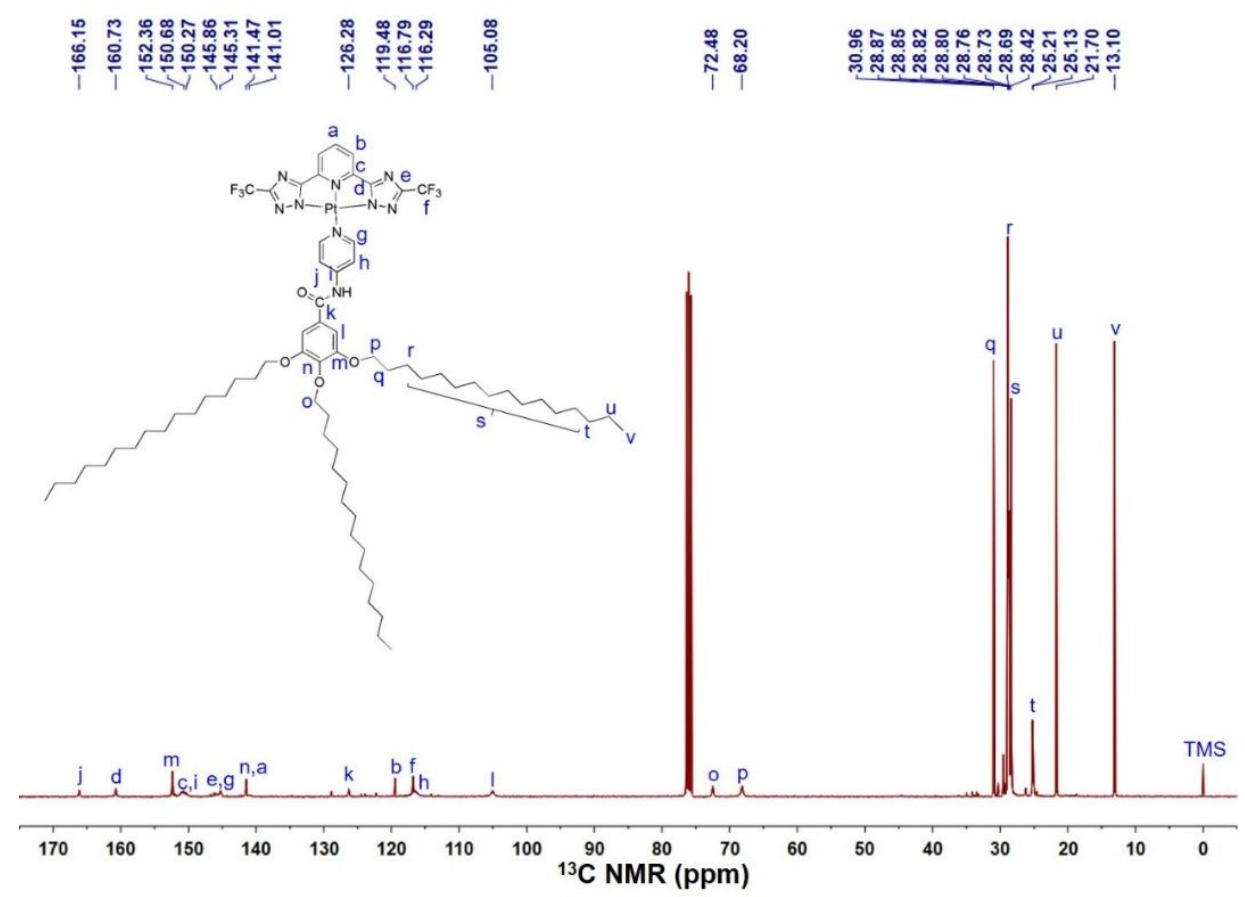

Figure S23. ${ }^{13} \mathrm{C}-\mathrm{NMR}$ spectrum of the complex $\mathbf{P t}-\mathbf{3 O C}_{\mathbf{1 6}} \mathbf{H}_{\mathbf{3 3}}$ in $\mathrm{CDCl}_{3}$

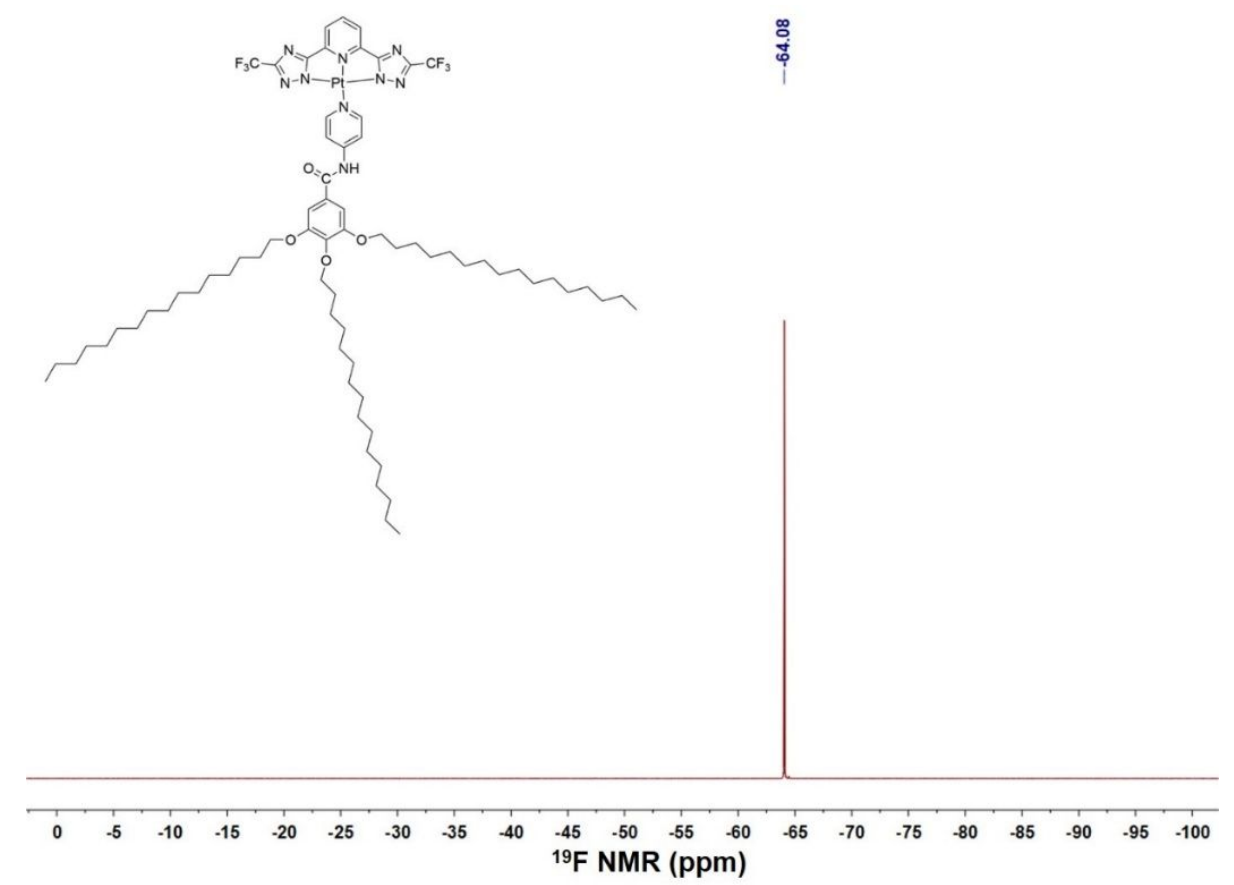

Figure S24. ${ }^{19} \mathrm{~F}-\mathrm{NMR}$ spectrum of the complex $\mathbf{P t}-\mathbf{3 O C} \mathbf{C}_{\mathbf{1 6}} \mathbf{H}_{\mathbf{3 3}}$ in $\mathrm{CDCl}_{3}$ 


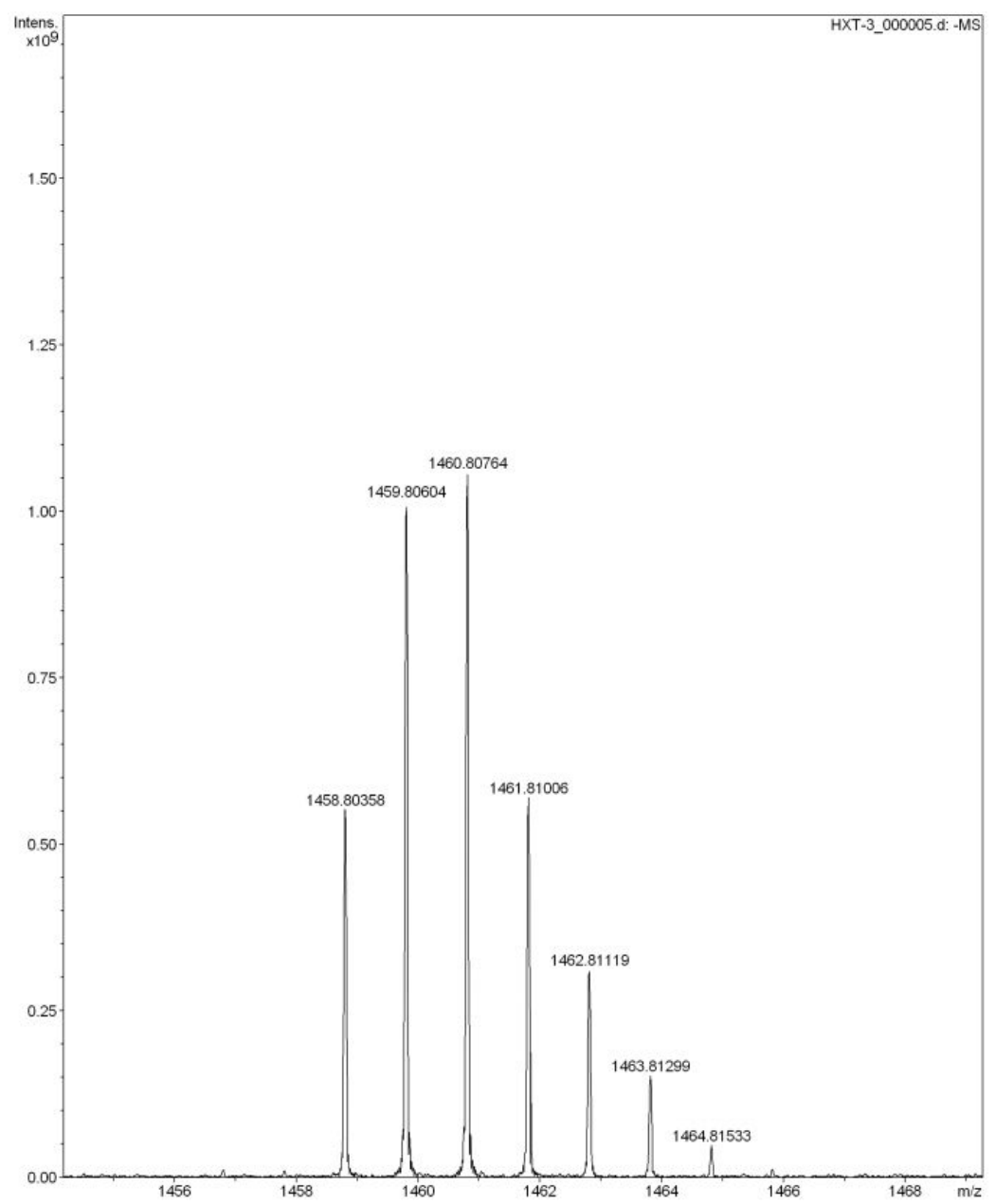

Figure S25. ESI-HR-MS of the complex Pt-3OC $\mathbf{1 0}_{\mathbf{1 6}} \mathbf{H}_{33}$

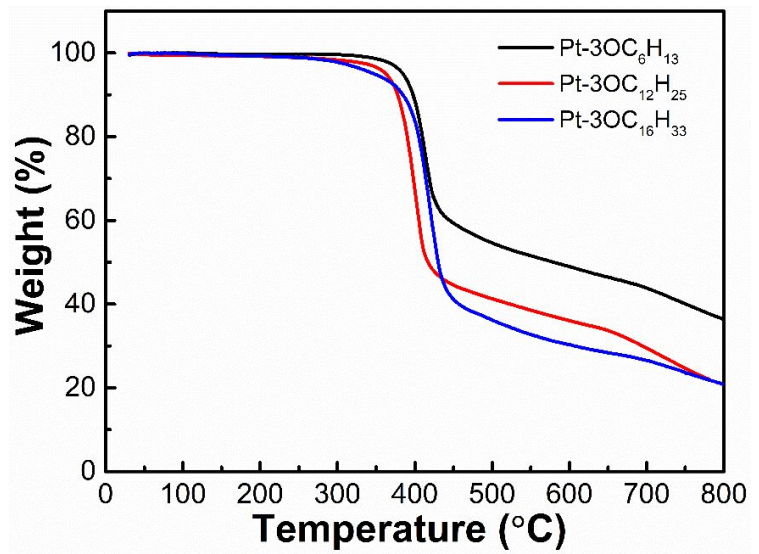

Figure S26. TGA curves of the three complexes at a heating rate of $10{ }^{\circ} \mathrm{C} / \mathrm{min}$ 

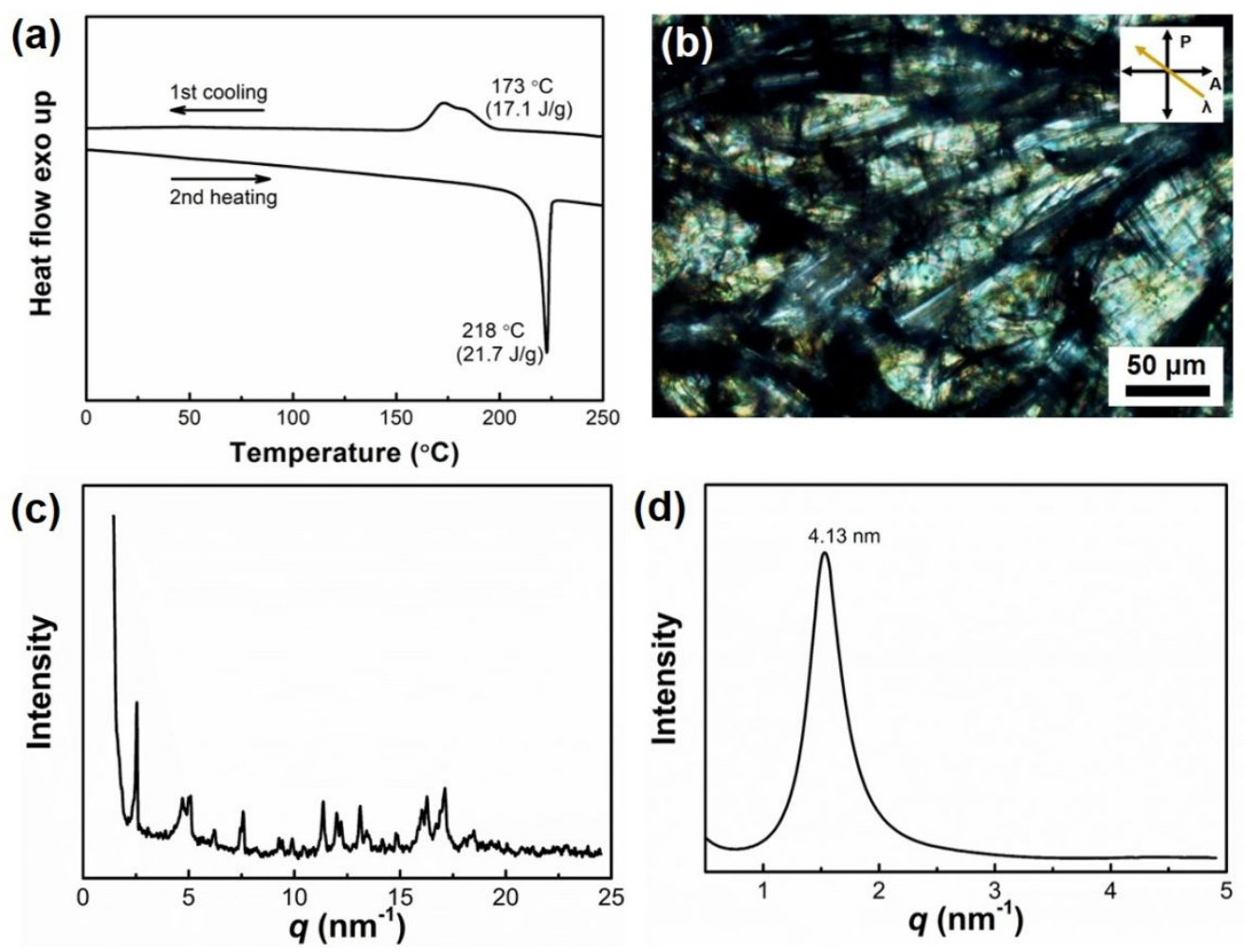

Figure S27. (a) DSC curves, (b) POM textures, (c) 1D-WAXD pattern after $1^{\text {st }}$ cooling, and (d) SAXS pattern of $\mathbf{P t - 3 O \mathbf { C } _ { 6 }} \mathbf{H}_{13}$ in powder at room temperature

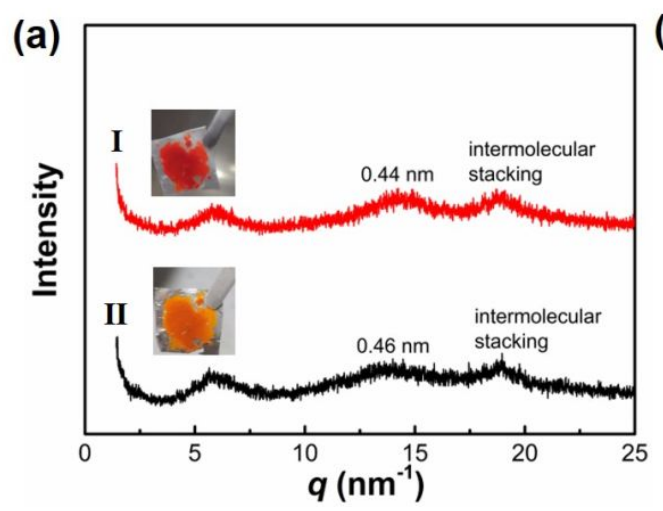

(b)

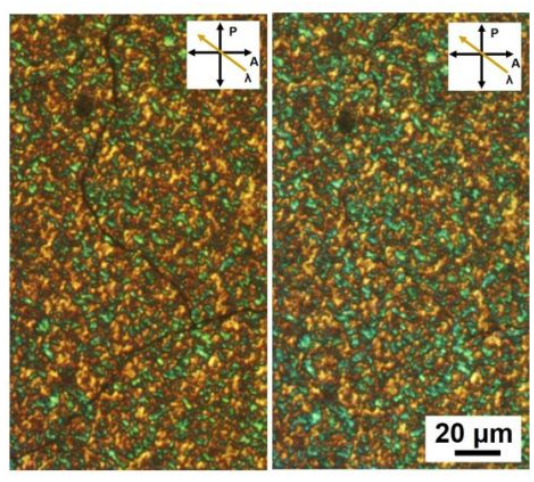

Figure S28. (a) 1D-WAXD patterns of $\mathbf{P t}-\mathbf{3 O C}_{\mathbf{1 6}} \mathbf{H}_{\mathbf{3 3}}$ when frozen by liquid nitrogen ( I ) and at room temperature (II), respectively; (b) POM images when frozen by liquid nitrogen (left) and at room temperature (right), respectively. 


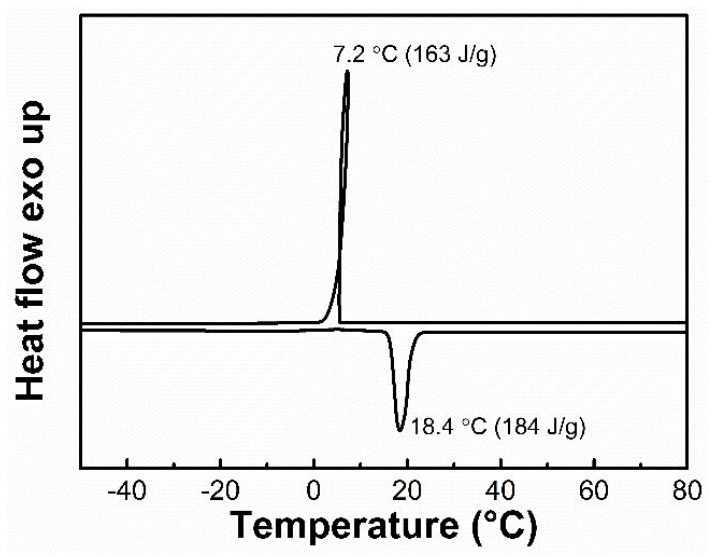

Figure S29. DSC curves of bromohexadecane

(a)

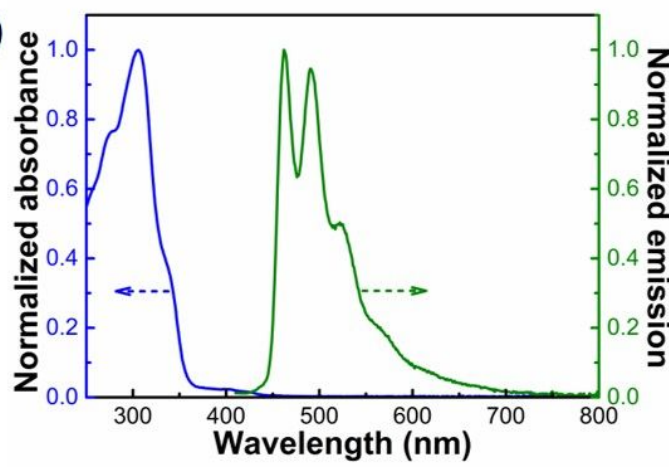

(b)

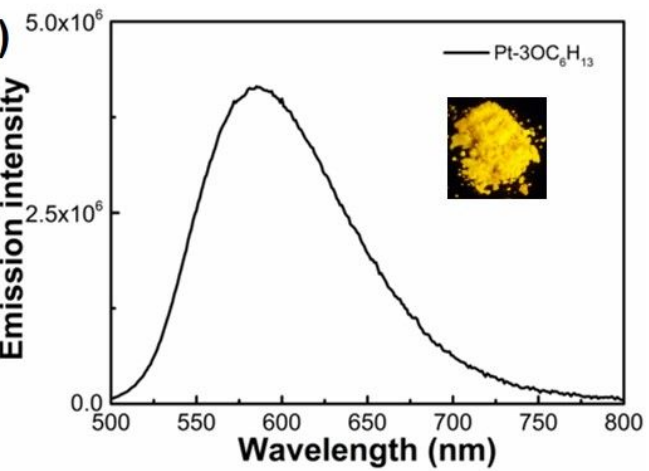

Figure S30. (a) Absorption and emission spectra of Pt-3OC $\mathbf{C}_{6} \mathbf{H}_{\mathbf{1 3}}$ in $\mathrm{CH}_{2} \mathrm{Cl}_{2}$ (concentration: $10^{-5} \mathrm{M}$ ) at room temperature; (b) Emission spectrum of $\mathbf{P t - 3 0 \mathbf { C } _ { 6 }} \mathbf{H}_{\mathbf{1 3}}$ in solid powder. Inset: Powder emission pictures under UV light (wavelength: $365 \mathrm{~nm}$ ). 

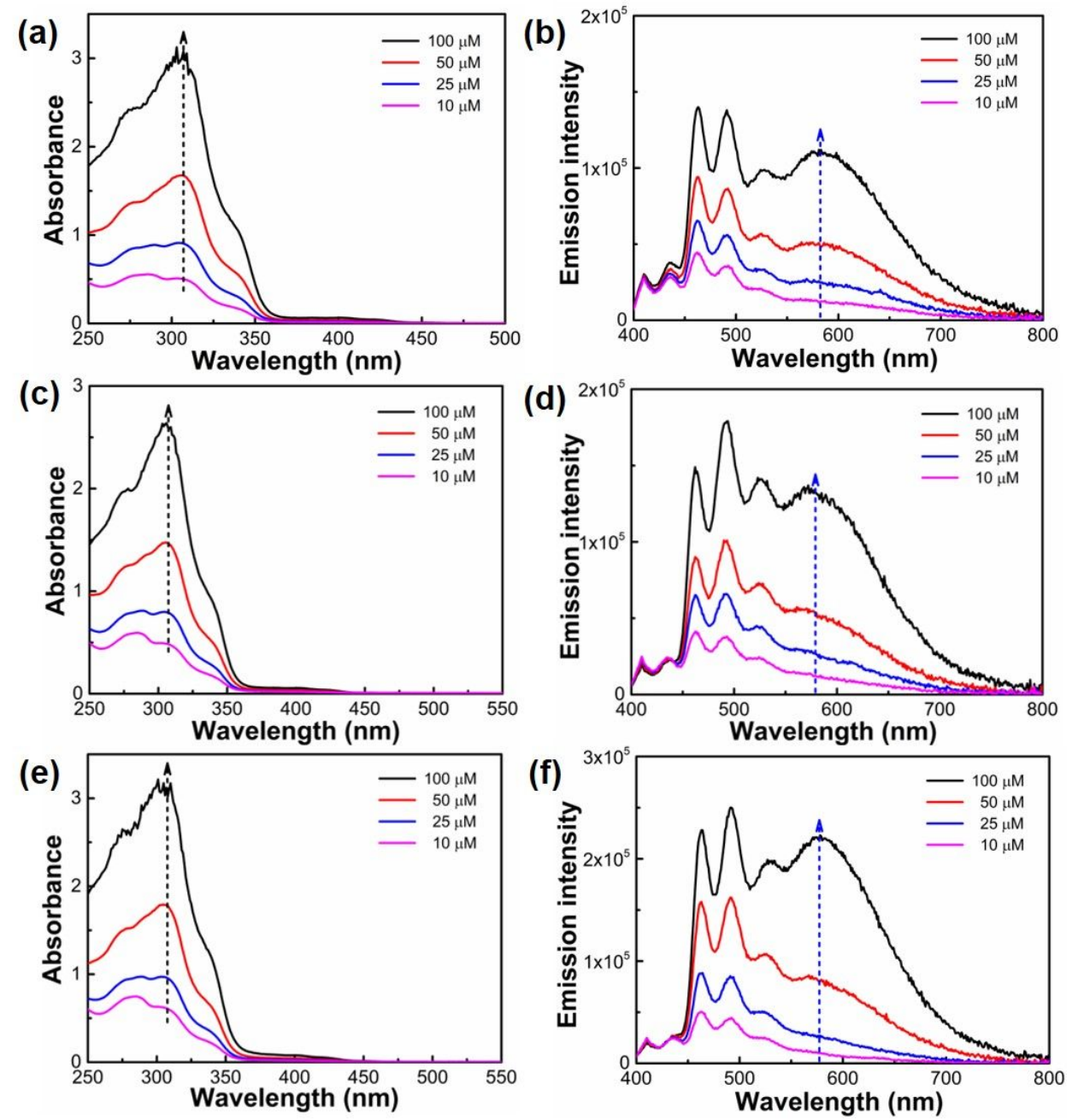

Figure S31. Absorption and emission spectra of (a, b) Pt-30C $\mathbf{C H}_{\mathbf{6}}$, (c, d) Pt-3OC $\mathbf{C O}_{\mathbf{1 2}} \mathbf{H}_{25}$ and (e, f) $\mathbf{P t}-\mathbf{3 O C} \mathbf{C}_{\mathbf{1 6}} \mathrm{H}_{33}$ at different concentrations in $\mathrm{CH}_{2} \mathrm{Cl}_{2}$. Excitation wavelength: $365 \mathrm{~nm}$. 

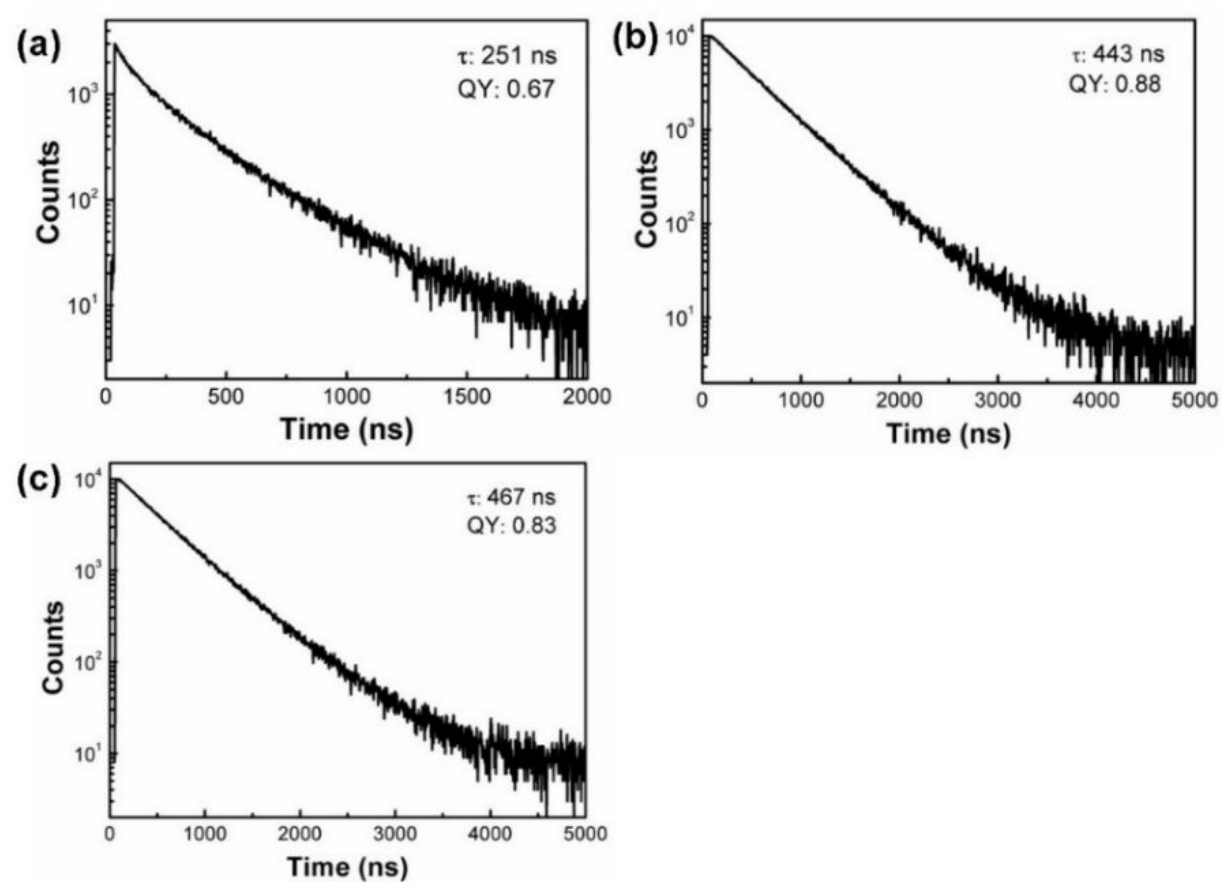

Figure S32. Emission decay of the bulky complexes: (a) $\mathbf{P t}-\mathbf{3 O C} \mathbf{C}_{\mathbf{6}} \mathbf{H}_{\mathbf{1 3}}$; (b) Pt$\mathbf{3 O C}_{12} \mathbf{H}_{25}$; (c) Pt-3OC $\mathbf{1 6}_{\mathbf{1 6}} \mathbf{H}_{33}$. : lifetime, QY: quantum yield.

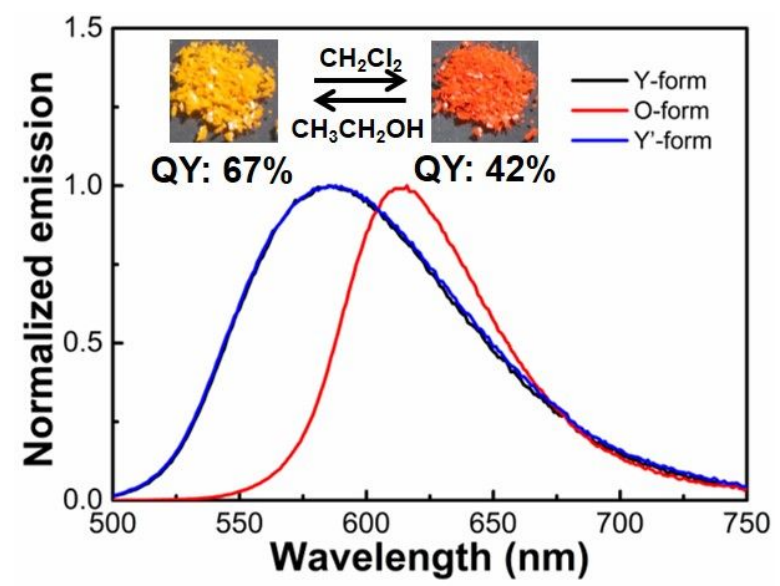

Figure S33. Emission spectra of $\mathbf{P t}-\mathbf{3 O C} \mathbf{C}_{6} \mathbf{H}_{13}$ in different forms when fuming with $\mathrm{CH}_{2} \mathrm{Cl}_{2}$ and $\mathrm{EtOH}$, respectively, excitation wavelength: $365 \mathrm{~nm}$. Inset: Color change under ambient light during the fuming process. 

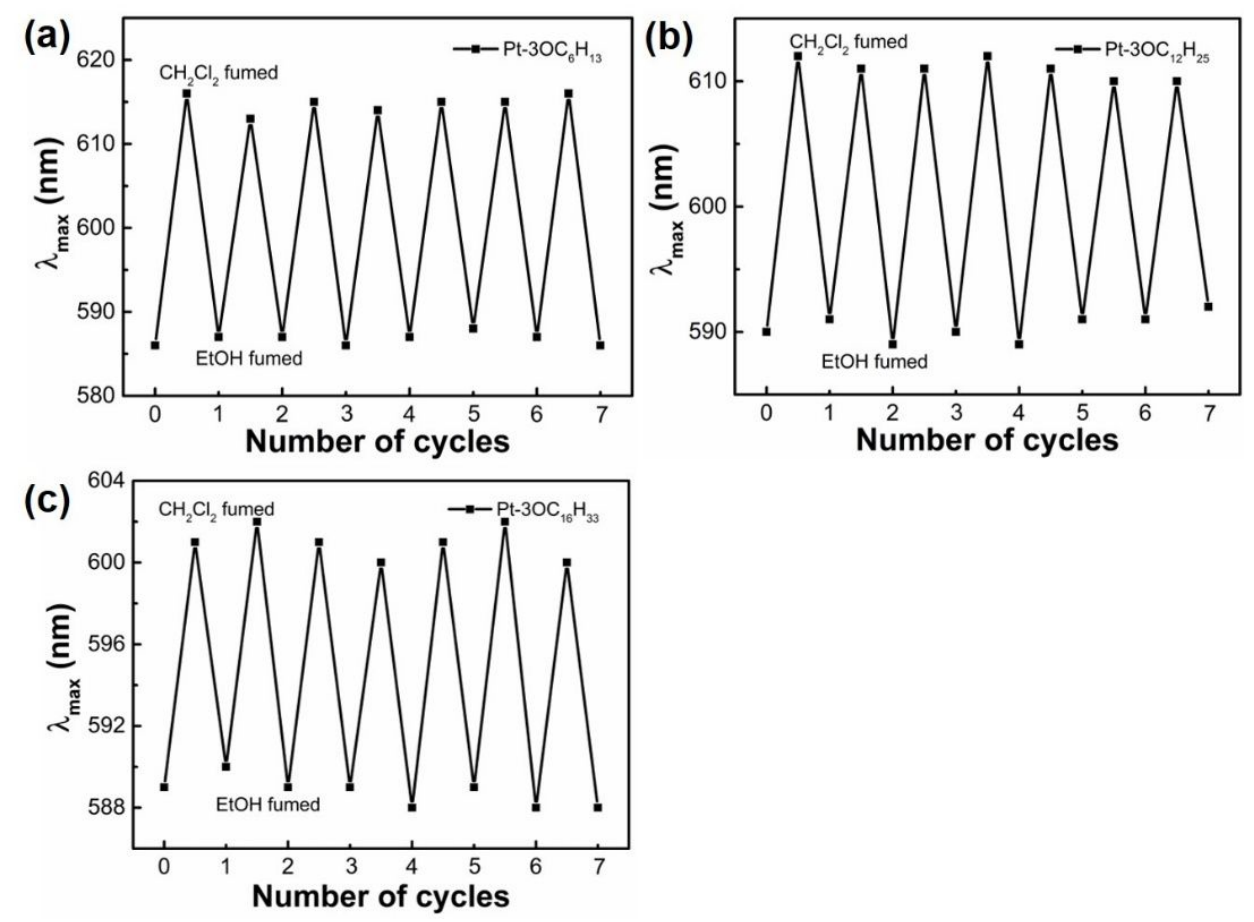

Figure S34. Reversible emission of (a) $\mathbf{P t}-\mathbf{3 O C}_{6} \mathbf{H}_{13}$, (b) Pt-3OC $\mathbf{1 2}_{\mathbf{1 2}} \mathbf{H}_{25}$ and (c) Pt$\mathbf{3 O C}_{\mathbf{1 6}} \mathrm{H}_{33}$ under alternative $\mathrm{CH}_{2} \mathrm{Cl}_{2}$ and $\mathrm{EtOH}$ vapor fuming. Excitation wavelength: $365 \mathrm{~nm}$.
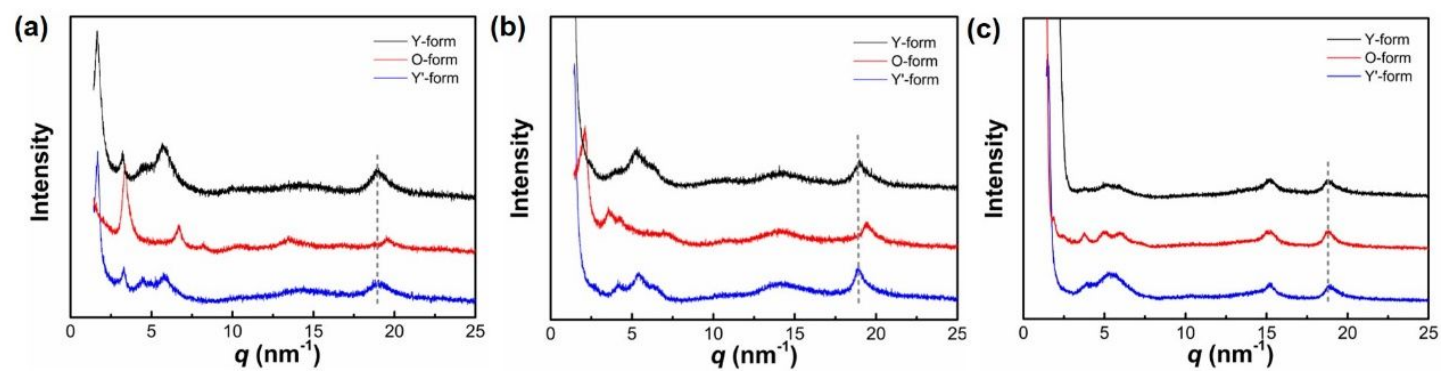

Figure S35. Powder 1D-WAXD patterns of (a) $\mathbf{P t - 3 O C} \mathbf{C H}_{\mathbf{1 3}}$, (b) $\mathbf{P t}-\mathbf{3 O C} \mathbf{C O}_{\mathbf{1 2}} \mathbf{H}_{25}$ and (c) Pt-3OC ${ }_{16} \mathbf{H}_{33}$ in their Y-form, O-form and $\mathrm{Y}^{\prime}$-form, respectively.

(a)

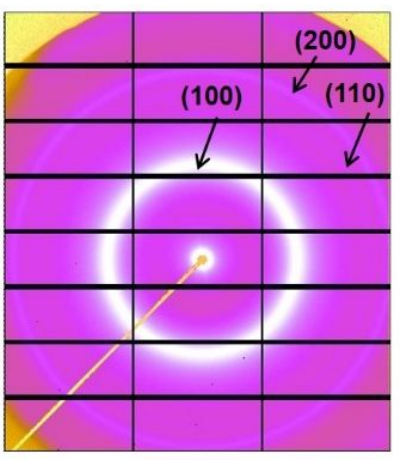

(b)

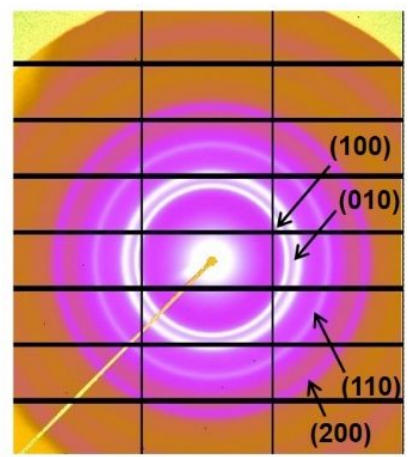

Figure S36. 2D SAXS patterns of (a) $\mathbf{P t}-\mathbf{3 O C} \mathbf{C}_{\mathbf{1 2}} \mathbf{H}_{\mathbf{2 5}}$ and (b) $\mathbf{P t}-\mathbf{3 O C} \mathbf{1 6}_{\mathbf{1 6}} \mathbf{H}_{\mathbf{3 3}}$ 
Table S1. Reported absolute quantum yields of LLCs in the solid state

\begin{tabular}{|c|c|c|}
\hline Chemical & Quantum yield & References \\
\hline TPE-PPE & $22 \%$ & 1 \\
\hline PAHs & $19 \%$ & 2 \\
\hline TPE-2PBN & $83 \%$ & 3 \\
\hline TPEMes & $55 \%$ & 4 \\
\hline Cationic Pt(II) complex & $86 \%$ & 5 \\
\hline Tridentate $N, C, N$-coordinated $\mathrm{Pt}(\mathrm{II})$ complexes & $12 \%$ & 6 \\
\hline Unsymmetrically four-coordinated Pt(II) complexes & $20 \%$ & 7 \\
\hline G-form of anthracene derivatives & $36 \%$ & \multirow{2}{*}{8} \\
\hline Y-form of anthracene derivatives & $25 \%$ & \\
\hline G-form of cyclophane & $54 \%$ & \multirow{2}{*}{9} \\
\hline B-form of cyclophane & $15 \%$ & \\
\hline FOEB & $9 \%$ & 10 \\
\hline $\mathrm{C}_{6} \mathrm{Ph}-\mathrm{HBT}$ & $39 \%$ & 11 \\
\hline Gold(I) complexes & $18 \%$ & 12 \\
\hline Anthracene derivatives & $25 \%$ & 13 \\
\hline SCLCPs & $17 \%$ & 14 \\
\hline Asymmetrical bis(pyrazolate) $\mathrm{Pt}(\mathrm{II})$ complexes & $20 \%$ & 15 \\
\hline Triphenylborane & $61 \%$ & 16 \\
\hline Phospholium species & $30 \%$ & 17 \\
\hline TPE polymer & $64 \%$ & 18 \\
\hline $\mathrm{PTPEC}_{4}$ & $35 \%$ & 19 \\
\hline TPE-DOAB & $46 \%$ & 20 \\
\hline TPE polymer & $52 \%$ & 21 \\
\hline G-DCS & $45 \%$ & 22 \\
\hline Z-CNBP & $28 \%$ & 23 \\
\hline GCS & $22 \%$ & 24 \\
\hline FD14 & $43 \%$ & 25 \\
\hline Oligo(p-phenylenevinylene) & $25 \%$ & 26 \\
\hline
\end{tabular}




\section{References}

(1) Zhao D., Fan F., Cheng J., Zhang Y., Wong K. S., Chigrinov V. G., Kwok H. S., Guo L., Tang B. Z. Light-Emitting Liquid Crystal Displays Based on an Aggregation-Induced Emission Luminogen. Adv. Opt. Mater., 2015, 3(2): 199202.

(2) Yuan W., Ren X. K., Li M., Guo H., Han Y., Wu M., Wang Q., Li M., Chen Y. From $S, N$-Heteroacene to Large Discotic Polycyclic Aromatic Hydrocarbons (PAHs): Liquid Crystal Versus Plastic Crystalline Materials with Tunable Mechanochromic Fluorescence. Angew. Chem. Int. Ed., 2018, 57(21): 61616165.

(3) Wang Y., Liao Y., Cabry C. P., Zhou D., Xie G., Qu Z., Bruce D. W., Zhu W. Highly Efficient Blueish-Green Fluorescent OLEDs Based on AIE Liquid Crystal Molecules: From Ingenious Molecular Design to Multifunction Materials. J. Mater. Chem. C, 2017, 5(16): 3999-4008.

(4) Liu Y., You L. H., Lin F. X., Fu K., Yuan W. Z., Chen E. Q., Yu Z. Q., Tang B. Z. Highly Efficient Luminescent Liquid Crystal with Aggregation-Induced Energy Transfer. ACS Appl. Mater. Interfaces, 2019, 11(3): 3516-3523.

(5) Krikorian M., Liu S., Swager T. M. Columnar Liquid Crystallinity and Mechanochromism in Cationic Platinum(II) Complexes. J. Am. Chem. Soc., 2014, 136(8): 2952-2955.

(6) Kozhevnikov V. N., Donnio B., Bruce D. W. Phosphorescent, Terdentate, Liquid-Crystalline Complexes of Platinum(II): Stimulus-Dependent Emission. Angew. Chem. Int. Ed., 2008, 47(33): 6286-6289.

(7) Cuerva C., Campo J. A., Cano M., Lodeiro C. Multi-Stimuli-Responsive Properties of Aggregation-Enhanced Emission-Active Unsymmetrical Pt(II) Metallomesogens through Self-Assembly. Chem. Eur. J., 2019, 25(52): 1204612051.

(8) Mase K., Sasaki Y., Sagara Y., Tamaoki N., Weder C., Yanai N., Kimizuka N. Stimuli-Responsive Dual-Color Photon Upconversion: A Singlet-to-Triplet Absorption Sensitizer in a Soft Luminescent Cyclophane. Angew. Chem. Int. 
Ed., 2018, 57(11): 2806-2810.

(9) Sagara Y., Weder C., Tamaoki N. Asymmetric Cyclophanes Permit Access to Supercooled Nematic Liquid Crystals with Stimulus-Responsive Luminescence. Chem. Mater., 2017, 29(14): 6145-6152.

(10) Tong J., Wang Y. J., Wang Z., Sun J. Z., Tang B. Z. Crystallization-Induced Emission Enhancement of a Simple Tolane-Based Mesogenic Luminogen. $J$. Phys. Chem. C, 2015, 119(38): 21875-21881.

(11) Zhang W., Sakurai T., Aotani M., Watanabe G., Yoshida H., Padalkar V. S., Tsutsui Y., Sakamaki D., Ozaki M., Seki S. Highly Fluorescent Liquid Crystals from Excited-State Intramolecular Proton Transfer Molecules. Adv. Opt. Mater., 2019, 7(2): 1801349 .

(12) Fujisawa K., Okuda Y., Izumi Y., Nagamatsu A., Rokusha Y., Sadaike Y., Tsutsumi O. Reversible Thermal-Mode Control of Luminescence from LiquidCrystalline Gold(I) Complexes. J. Mater. Chem. C, 2014, 2(18): 3549-3555.

(13) Yamane S., Sagara Y., Mutai T., Araki K., Kato T. Mechanochromic Luminescent Liquid Crystals Based on a Bianthryl Moiety. J. Mater. Chem. C, 2013, 1(15): 2648-2656.

(14) Yuan Y., Li J., He L., Liu Y., Zhang H. Preparation and Properties of Side Chain Liquid Crystalline Polymers with Aggregation-Induced Emission Enhancement Characteristics. J. Mater. Chem. C, 2018, 6(26): 7119-7127.

(15) Cuerva C., Campo J. A., Cano M., Schmidt R., Lodeiro C. Multifunctional Pt(II) Metallomesogens Exhibiting Luminescence and Proton Conductivity in the Mesophase near Room Temperature. J. Mater. Chem. C, 2018, 6(36): 97239733.

(16) Kushida T., Shuto A., Yoshio M., Kato T., Yamaguchi S. A Planarized Triphenylborane Mesogen: Discotic Liquid Crystals with Ambipolar ChargeCarrier Transport Properties. Angew. Chem. Int. Ed., 2015, 54(23): 6922-6925.

(17) Ren Y., Kan W. H., Henderson M. A., Bomben P. G., Berlinguette C. P., Thangadurai V., Baumgartner T. External-Stimuli Responsive Photophysics and Liquid Crystal Properties of Self-Assembled "Phosphole-Lipids". J. Am. 
Chem. Soc., 2011, 133(42): 17014-17026.

(18) Yuan W. Z., Yu Z.-Q., Tang Y., Lam J. W. Y., Xie N., Lu P., Chen E.-Q., Tang B. Z. High Solid-State Efficiency Fluorescent Main Chain Liquid Crystalline Polytriazoles with Aggregation-Induced Emission Characteristics. Macromolecules, 2011, 44(24): 9618-9628.

(19) Tao L., Li M. L., Yang K. P., Guan Y., Wang P., Shen Z., Xie H. L. ColorTunable and Stimulus-Responsive Luminescent Liquid Crystalline Polymers Fabricated by Hydrogen Bonding. ACS Appl. Mater. Interfaces, 2019, 11(16): 15051-15059.

(20) Jing H., Lu L., Feng Y., Zheng J.-F., Deng L., Chen E.-Q., Ren X.-K. Synthesis, Aggregation-Induced Emission, and Liquid Crystalline Structure of Tetraphenylethylene-Surfactant Complex Via Ionic Self-Assembly. J. Phys. Chem. C, 2016, 120(48): 27577-27586.

(21) Wang Y., Liao Y., Cabry C. P., Zhou D., Xie G., Qu Z., Bruce D. W., Zhu W. Highly Efficient Blueish-Green Fluorescent OLEDs Based on AIE Liquid Crystal Molecules: From Ingenious Molecular Design to Multifunction Materials. J. Mater. Chem. C, 2017, 5(16): 3999-4008.

(22) Yoon S.-J., Kim J. H., Kim K. S., Chung J. W., Heinrich B., Mathevet F., Kim P., Donnio B., Attias A.-J., Kim D., Park S. Y. Mesomorphic Organization and Thermochromic Luminescence of Dicyanodistyrylbenzene-Based Phasmidic Molecular Disks: Uniaxially Aligned Hexagonal Columnar Liquid Crystals at Room Temperature with Enhanced Fluorescence Emission and Semiconductivity. Adv. Funct. Mater., 2012, 22(1): 61-69.

(23) Zhu Y., Zheng M., Tu Y., Chen X.-F. Supramolecular Fluorescent Polymers Containing A-Cyanostilbene-Based Stereoisomers: Z/E-Isomerization Induced Multiple Reversible Switching. Macromolecules, 2018, 51(9): 3487-3496.

(24) Park J. W., Nagano S., Yoon S. J., Dohi T., Seo J., Seki T., Park S. Y. High Contrast Fluorescence Patterning in Cyanostilbene-Based Crystalline Thin Films: Crystallization-Induced Mass Flow Via a Photo-Triggered Phase Transition. Adv. Mater., 2014, 26(9): 1354-1359. 
(25) Martínez-Abadía M., Varghese S., Romero P., Gierschner J., Giménez R., Ros M. B. Highly Light-Sensitive Luminescent Cyanostilbene Flexible Dimers. $A d v$. Opt. Mater., 2017, 5(4): 1600860.

(26) Yagai S., Okamura S., Nakano Y., Yamauchi M., Kishikawa K., Karatsu T., Kitamura A., Ueno A., Kuzuhara D., Yamada H., Seki T., Ito H. Design Amphiphilic Dipolar $\pi$-Systems for Stimuli-Responsive Luminescent Materials Using Metastable States. Nat. Commun., 2014, 5: 4013. 